\title{
Spin transition of an iron(II) organoborate complex in different polymorphs and in vacuum-deposited thin films: Influence of cooperativity
}

\section{Supporting Information}

Sascha Ossinger ${ }^{a}$, Christian Näther $^{a *}$, Axel Buchholz ${ }^{b}$, Marc Schmidtmann $^{c}$, Sebastian Mangelsen ${ }^{a}$, Rüdiger Beckhaus ${ }^{c}$, Winfried Plass ${ }^{b}$ and Felix Tuczek ${ }^{a *}$

*Institute of Inorganic Chemistry, Christian-Albrechts-University Kiel, Max-Eyth-Str.2, 24118 Kiel, Germany

\# Institute of Inorganic and Analytical Chemistry, Friedrich-Schiller-University, 07743 Jena, Germany

+ Institute for Chemistry, Carl von Ossietzky University Oldenburg, 26111 Oldenburg, Germany

\section{Table of Content}

\section{Experimental Section}

Synthesis of all compounds (1-4) 3

$\begin{array}{lll}\text { Fig. S1 Experimental powder pattern, Pawley fit and difference plot of 1-II. } & 8\end{array}$

Fig. S2 Experimental powder pattern, Pawley fit and difference plot of 1-I. $\quad 9$

Fig. S3 Experimental powder pattern, Pawley fit and difference plot of 2 . 9

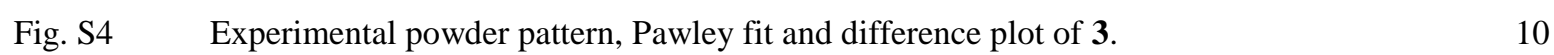

Table S1 Crystal data and details of the structure refinement for 1-II, 2 and 3-MeOH at $200 \mathrm{~K} . \quad 11$

Table S2 Crystal data and details of the structure refinement for 1-II at 300, 393, $423 \mathrm{~K}$ and $300 \mathrm{~K} 12$ after cooling from $393 \mathrm{~K}$.

\section{Thermoanalytical measurements}

Fig. S5 Experimental XRPD pattern of the residue obtained by heating to $155^{\circ} \mathrm{C}$ and cooling of 1- 13 II and calculated pattern of 1-II.

Fig. S6 Heating and cooling cycles for 1-II at $10^{\circ} \mathrm{C} / \mathrm{min}$. 13

Fig. S7 Heating and cooling cycles for 1-II at $10^{\circ} \mathrm{C} / \mathrm{min}$ between 25 and $160{ }^{\circ} \mathrm{C}$ with annealing the 14 sample at $160{ }^{\circ} \mathrm{C}$ for $1 \mathrm{~h}$ after each cycle.

Fig. S8 Experimental XRPD pattern 1-I prepared in a DSC measurement, calculated pattern for the 14 Zn compound $\mathbf{2}$ and calculated pattern of the Co compound $\mathbf{3}$.

Fig. S9 DSC curves of 1-II at different heating rates. $\quad 15$

Fig. S10 Microscopic images of 1-I and 1-II at different temperatures. 16

Fig. S11 DSC curve of $\mathbf{1 - I}$ at $10{ }^{\circ} \mathrm{C} / \mathrm{min}$. 17 
Fig. S12 Experimental XRPD pattern of the residue obtained after the exothermic peak observed in an DSC measurement of 1-I.

Fig. S13 Selected XRPD patterns of 1-II measured at different temperatures.

Fig. S14 Experimental XRPD pattern of the residue obtained by heating of 1-II to $171{ }^{\circ} \mathrm{C}$ resulting 18 in 1-I measured at room temperature and calculated pattern of 1-I.

Fig. S15 DSC curve of form $\mathbf{1 - I I}$ at $10^{\circ} \mathrm{C} / \mathrm{min}$ from 25 to $160{ }^{\circ} \mathrm{C}$ and at $0.1{ }^{\circ} \mathrm{C} / \mathrm{min}$ from 160 to 23018 ${ }^{\circ} \mathrm{C}$.

Fig. S16 Experimental XRPD pattern of the residue obtained after the exothermic event at $178^{\circ} \mathrm{C}$ observed in an DSC measurement of $\mathbf{1 - I I}$ and calculated pattern for the $\mathrm{Zn}$ compound $\mathbf{2}$ and of the Co compound 3 .

Fig. S17 Semi-empirical energy-temperature diagram showing the thermodynamic relations between 1-I and 1-II.

Fig. S18 Pawley fits of powder patterns of 1-I at measured at $171^{\circ} \mathrm{C}$ and of $\mathbf{1 - I I}$ measured at $159^{\circ} \mathrm{C} . \quad 20$

Fig. S19 DSC heating and cooling curve of 2 and 3 at $10^{\circ} \mathrm{C} / \mathrm{min}$ from 25 to $300{ }^{\circ} \mathrm{C}$.

\section{Magnetic Susceptibility and Mößbauer Spectroscopic Measurements}

Fig. S20 $\quad \chi_{M} T$ vs $T$ curves from PPMS measurements of compound 3 between 2 and $300 \mathrm{~K}$.

\section{Vibrational spectroscopy}

Fig. S21 Selected and simplified metal-ligand stretching vibrations calculated for 1.

Table S3 Selected Far-Infrared vibrational and Resonance-Raman frequencies [ $\mathrm{cm}^{-1}$ ] for 1-II and 1- 21 I.

\section{Investigations of vacuum-deposited films}

Fig. S22 Fourier transform infrared (FT-IR) spectra of bulk material and vacuum-deposited material of $\mathbf{2}$ and $\mathbf{3}$ at $298 \mathrm{~K}$.

Fig. S23 Experimental X-ray-diffractograms of film on glass for $\mathbf{1}$.

\section{Crystal structures and temperature dependent single crystal measurements}

Fig. S24 View of the asymmetric unit of 1-II, 2 and 3-MeOH.

Table S4. Selected bond lengths [ $\quad$ Å] and angles $\left[{ }^{\circ}\right]$ for $\mathbf{1 - I I}$ at 300, 393, $423 \mathrm{~K}$ and $300 \mathrm{~K}$ after cooling from $393 \mathrm{~K}$.

Table S5 Selected bond lengths $[\AA]$ and angles $\left[{ }^{\circ}\right]$ for 2 and 3-MeOH at $200 \mathrm{~K}$.

Table S6 Structural parameters $\Sigma\left[{ }^{\circ}\right]$ and $\theta\left[{ }^{\circ}\right]$ used to characterize the distortion of the FeN 624 octahedron for 1-II at 200, 303, 393 and $423 \mathrm{~K}, \mathbf{2}$ and 3-MeOH at $200 \mathrm{~K}$.

Fig. S25 Crystal structure of $\mathbf{2}$ that is isotypic to 1-I1- with view along the crystallographic $b$-axis. 25

Fig. S26 $\quad \gamma_{H S}$ plot vs. $T$ obtained from temperature-dependent single crystal measurement of 1-II. 25

Table S7 Temperature-dependent unit cell parameter for 1-II. 26

\section{Coordinates from the DFT calculations of 1}




\section{Experimental Section}

All reactions were carried out in dry solvents and the complexation was carried out under nitrogen-atmosphere using standard Schlenk techniques or in a M. Braun Labmaster 130 glovebox under argon.

1H-Pyrazole, 2-(1H-Pyrazol-3-yl)pyridine, Potassium tetrahydroborate, zinc(II) and cobalt(II) triflate were purchased from commercial sources and used without further purification. Iron(II) triflate is also commercial available but was purified by following method: The compound was solved in dry methanol (a few $\mathrm{mL}$ for a supersaturated solution), filtered off and afterwards the solvent was removed in vacuum. Solvents were purchased from commercial sources and purified by distilling over conventional drying agents.

Synthesis of $\mathbf{K}\left[\mathbf{H}_{2} \mathbf{B}(\mathbf{p z})(\mathbf{p y p z})\right]$ (4): Potassium tetrahydroborate (540 mg, $\left.0.01 \mathrm{~mol}\right)$, 1H-Pyrazole (681 mg, $0.01 \mathrm{~mol})$ and 2-(1H-Pyrazol-3-yl)pyridine (1.45 g, $0.01 \mathrm{~mol})$ were suspended in toluene $(20 \mathrm{~mL})$ and refluxed for $17 \mathrm{~h}$. Upon cooling the filtrate to room temperature, a viscous substance formed that was filtered off. Afterwards a few hours later a white precipitate formed and after one additionally night of crystallisation the precipitate were collected by suction filtration. Yield $555 \mathrm{mg}\left(2.11 \mathrm{mmol}, 21 \%\right.$ vs. $\left.\mathrm{K}\left[\mathrm{BH}_{4}\right]\right)$. If necessary, it is possible to recrystallize the compound in toluene, which affords the ligand free of any contaminants.

Elemental analysis calculated for $\mathrm{C}_{11} \mathrm{H}_{11} \mathrm{BKN}_{5}$ : C 50.21, H 4.21, N $26.61 \%$, found: C 50.37, H 4.32, N $26.32 \%$.

HRESI-MS $(+)\left(\mathbf{C H}_{3} \mathbf{C N}\right): m / z(\%)=[\mathrm{M}-\mathrm{K}+2 \mathrm{H}]^{+}$calcd. 226.12585, found 226.12571 (2); $[\mathrm{M}+\mathrm{H}]^{+}$calcd. 264.08173, found $264.08154(12) ;[\mathrm{M}+\mathrm{K}]^{+}$calcd. 302.03762, found 302.03734 (4).

HRESI-MS(-) $\left(\mathbf{C H}_{3} \mathbf{C N}\right): \mathrm{m} / z(\%)=[\mathbf{M}-\mathrm{K}]^{-}$calcd. 224.113, found 224.11071 (26).

${ }^{1} \mathbf{H}$ NMR (500 MHz, CD $\left.\mathbf{C N}\right): \delta / \mathrm{ppm}=8.51(\mathrm{ddd}, \mathrm{J}=4.9 \mathrm{~Hz}, 1.8 \mathrm{~Hz}, 1.0 \mathrm{~Hz}, 1 \mathrm{H}$, pyridyl- $\mathrm{H}^{8}$ or $\left.\mathrm{H}^{7}\right), 7.72\left(\mathrm{~m}, 2 \mathrm{H}\right.$, pyridyl- $\mathrm{H}^{10}$ and $\mathrm{H}^{8}$ or $\left.\mathrm{H}^{7}\right), 7.50(\mathrm{~d}, \mathrm{~J}=2.0 \mathrm{~Hz}, 1 \mathrm{H}$, pyrazolyl$\left.\mathrm{H}^{5}\right), 7.48\left(\mathrm{dd}, \mathrm{J}=2.0 \mathrm{~Hz}, 0.5 \mathrm{~Hz}, 1 \mathrm{H}\right.$, pyrazolyl- $\left.\mathrm{H}^{5 \mathrm{~A}}\right), 7.37\left(\mathrm{~d}, \mathrm{~J}=1.1 \mathrm{~Hz}, 1 \mathrm{H}\right.$, pyrazolyl- $\left.\mathrm{H}^{3 \mathrm{~A}}\right)$, $7.15\left(\mathrm{ddd}, \mathrm{J}=7.2 \mathrm{~Hz}, 4.9 \mathrm{~Hz}, 1.4 \mathrm{~Hz}, 1 \mathrm{H}\right.$, pyridyl- $\left.\mathrm{H}^{9}\right), 6.56\left(\mathrm{~d}, \mathrm{~J}=2.0 \mathrm{~Hz}, 1 \mathrm{H}\right.$, pyrazolyl- $\left.\mathrm{H}^{4}\right)$, $6.02\left(\mathrm{t}, \mathrm{J}=1.8 \mathrm{~Hz}, 1 \mathrm{H}\right.$, pyrazolyl-H $\left.{ }^{4 \mathrm{~A}}\right), 3.59$ (dd, J = $\left.192.0 \mathrm{~Hz}, 75.3 \mathrm{~Hz}, 2 \mathrm{H}, \mathrm{B}-\mathrm{H}\right)$.

${ }^{13} \mathbf{C}\left\{{ }^{1} \mathbf{H}\right\}$ NMR $\left(125 \mathrm{MHz}, \mathbf{C D}_{3} \mathrm{CN}\right): \delta / \mathrm{ppm}=154.67\left(\mathrm{C}_{\mathrm{q}}\right.$, pyridyl-C $\left.{ }^{6}\right), 151.86\left(\mathrm{C}_{\mathrm{q}}\right.$, pyrazolyl- $\left.\mathrm{C}^{3}\right), 150.24\left(\mathrm{CH}\right.$, pyridyl- $\mathrm{C}^{8}$ or $\left.\mathrm{C}^{7}\right), 139.64\left(\mathrm{CH}\right.$, pyrazolyl- $\left.\mathrm{C}^{3 \mathrm{~A}}\right), 137.51(\mathrm{CH}$, pyridyl-C $\mathrm{C}^{8}$ or $\left.\mathrm{C}^{7}\right), 136.49\left(\mathrm{CH}\right.$, pyrazolyl- $\left.\mathrm{C}^{5}\right), 135.05\left(\mathrm{CH}\right.$, pyrazolyl-C $\left.\mathrm{C}^{5 \mathrm{~A}}\right), 122.21(\mathrm{CH}$, 
pyridyl-C $\left.\mathrm{C}^{9}\right), 120.66\left(\mathrm{CH}\right.$, pyridyl- $\left.\mathrm{C}^{10}\right), 103.69\left(\mathrm{CH}\right.$, pyrzazolyl- $\left.\mathrm{C}^{4 \mathrm{~A}}\right), 102.95(\mathrm{CH}$, pyrazolyl$\mathrm{C}^{4}$ ).

${ }^{11}$ B NMR (160 MHz, CD3CN): $\delta / p p m=-6.94(t, J=97.9 \mathrm{~Hz}, 1 \mathrm{~B})$.

IR (ATR): $v / \mathrm{cm}^{-1}=3124,3107,3093,3048(\mathrm{w}, v[=\mathrm{C}-\mathrm{H}]), 2374,2350,2327$ (m, vasym. [$\left.\left.\mathrm{BH}_{2}\right]\right), 2264\left(\mathrm{~m}, v_{\text {sym. }}\left[-\mathrm{BH}_{2}\right]\right), 1725(\mathrm{w}), 1619(\mathrm{w}), 1592(\mathrm{~s}), 1562(\mathrm{~m}), 1515(\mathrm{w}), 1492(\mathrm{~m}), 1452$ (w), 1427 (s), 1392 (m), 1354 (m), 1282 (m), 1221 (m), 1188 (s), 1151 (s), 1125 (w), 1084 (m), 1062 (m), 1047 (s), 1013 (w), 992 (w), 960 (m), 915 (w), 898 (m), 884 (m), 872 (w), 796 (m), $768(\mathrm{~m}), 755(\mathrm{~s}), 745$ (s), $710(\mathrm{~m}), 698(\mathrm{~m}), 672(\mathrm{w}), 653(\mathrm{~m}), 631(\mathrm{~m}), 621(\mathrm{~m}), 525(\mathrm{w}), 465$ (w), $404(\mathrm{~m})$.

Raman (Bulk): $v / \mathrm{cm}^{-1}=3124,3107,3095,3072,3049,3011(\mathrm{~m}, v[=\mathrm{C}-\mathrm{H}]), 2378,2353$, $2332\left(\mathrm{w}, v_{\text {asym. }}\left[-\mathrm{BH}_{2}\right]\right), 2268\left(\mathrm{w}, v_{\text {sym. }}\left[-\mathrm{BH}_{2}\right]\right), 1595(\mathrm{~s}), 1564(\mathrm{~m}), 1518(\mathrm{~s}), 1493(\mathrm{~m}), 1452(\mathrm{w})$, 1394 (m), 1366 (s), 1283 (m), 1259 (w), 1242 (w), 1223 (w), 1202 (m), 1155 (w), 1084 (w), $1063(\mathrm{w}), 1053$ (w), 997 (s), 959 (m), 922 (w), 799 (w), $781(\mathrm{w}), 712(\mathrm{w}), 656(\mathrm{w}), 625(\mathrm{w})$, $525(\mathrm{w}), 467(\mathrm{w}), 407(\mathrm{w})$.

Synthesis of $\left[\mathbf{F e}\left\{\mathrm{H}_{2} \mathbf{B}(\mathbf{p z})(\mathbf{p y p z})\right\}_{2}\right]$ (1-II): To a solution of $\mathrm{Fe}(\mathrm{OTf})_{2} \quad(163 \mathrm{mg}$, $460 \mu \mathrm{mol})$ in methanol $(1 \mathrm{~mL})$ a solution of $\mathrm{K}\left[\mathrm{H}_{2} \mathrm{~B}(\mathrm{pz})(\mathrm{pypz})\right](242 \mathrm{mg}, 920 \mu \mathrm{mol})$ in methanol $(5 \mathrm{~mL})$ was added dropwise, leading to the formation of a red-brown colored solution. Immediately, a dark red colored precipitate was formed. The solution was stirred for one hour at room temperature and then the precipitate was filtered off, washed with methanol $(2 \times 5 \mathrm{~mL})$, dried under reduced pressure $(2.5 \mathrm{~h})$ and finally overnight under a stream of $\mathrm{N}_{2}$. Yield: $143 \mathrm{mg}$ $\left.(283 \mu \mathrm{mol}, 62 \% \text { vs. Fe(OTf })_{2}\right)$.

Elemental analysis calculated for $\mathrm{C}_{22} \mathrm{H}_{22} \mathrm{~B}_{2} \mathrm{FeN}_{10}$ : C 52.43, H 4.4, N $27.79 \%$, found: C 52.60, H 4.45, N $27.49 \%$.

HRESI-MS(+)(CH $\mathbf{C N}+\mathbf{M e O H}): m / z(\%)=\left[\mathrm{M}-\text { Ligand }+\mathrm{CH}_{3} \mathrm{CN}\right]^{+}$calcd. 321.07169, found 321.07154 (16); $\left[\mathrm{M}-\text { Ligand }+\mathrm{CH}_{3} \mathrm{CN}+\mathrm{MeOH}\right]^{+}$calcd. 353.09791, found 353.09772 (48); $[\mathrm{M}]^{+}$calcd. 504.15590, found $504.15599(65) ;[\mathrm{M}+\mathrm{H}]^{+}$calcd. 505.16372, found $505.16349(38) ;[\mathrm{M}+\mathrm{Na}]^{+}$calcd. 527.14566, found $527.14579(27) ;[\mathrm{M}+\mathrm{K}]^{+}$calcd. 543.11960; found 543.11959 (6).

${ }^{11} \mathrm{~B}$ NMR (128 MHz, CD3CN): $\delta / \mathrm{ppm}=25.16$ (br. s, 1B).

IR (ATR): $v / \mathrm{cm}^{-1}=3126,3115,3093,3069(\mathrm{w}, v[=\mathrm{C}-\mathrm{H}]), 2403,2391,2364,2334$ (m,

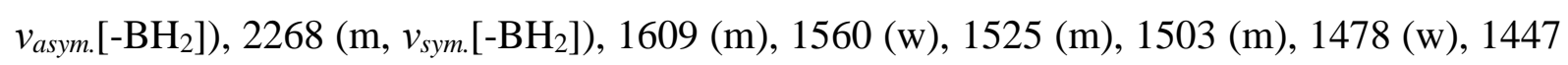
(m), 1435 (m), 1403 (m), 1388 (m), 1347 (m), 1288 (m), 1268 (m), 1247 (w), 1198 (s), 1184 (s), 1149 (s), 1135 (s), 1092 (m), 1076 (m), 1056 (s), 1011 (m), 988 (m), 964 (m), 919 (w), 898 
(m), $882(\mathrm{~s}), 874(\mathrm{~s}), 845(\mathrm{~m}), 784(\mathrm{w}), 749$ (s), $723(\mathrm{~s}), 694(\mathrm{~m}), 674(\mathrm{~m}), 641(\mathrm{~m}), 627(\mathrm{~m})$, $612(\mathrm{~m}), 514(\mathrm{w}), 500(\mathrm{~m}), 455(\mathrm{~m}), 441(\mathrm{~m})$.

Raman (Bulk): $v / \mathrm{cm}^{-1}=3136,3120,3070(\mathrm{~m}, v[=\mathrm{C}-\mathrm{H}]), 2405,2343\left(\mathrm{~m}, v_{\text {asym. }}\left[-\mathrm{BH}_{2}\right]\right)$, $2272\left(\mathrm{~m}, v_{\text {sym. }}\left[-\mathrm{BH}_{2}\right]\right), 1610$ (m), 1560 (m), 1526 (s), 1479 (m), 1450 (w), 1437 (w), 1408 (w), 1389 (w), 1348 (s), 1302 (w), 1277 (m), 1248 (w), 1202 (m), 1188 (m), 1159 (w), 1138 (w), 1096 (m), 1057 (w), 1016 (s), 964 (s), 920 (w), 704 (m), 455 (w).

UV/Vis (KBr): $\lambda_{\max } / \mathrm{nm}=$ 206, 244, 292, 398 (br), 465 (br), 518 (br).

The bulk material showed no visible color change with liquid nitrogen.

Single crystals of compound 1-II were obtained under nitrogen atmosphere by dissolving the microcrystalline compound obtained by synthesis in dry toluene and overlay this solution with dry $n$-hexane. After a few days dark red colored needle-like single crystals were obtained that were collected and dried under reduced pressure. To further characterize compound 1-II a single crystal structure determination has been performed The phase purity was confirmed by comparison of the experimental XRPD pattern with that calculated from single crystal data by Pawley fits (Figure S1).

Thin films can be prepared by physical vapour deposition under similar conditions ( $p=$ $10^{-2} \mathrm{mbar}, T=160{ }^{\circ} \mathrm{C}$ and $t=4 \mathrm{~min}$ ) as previously reported. ${ }^{1-6}$

Synthesis of [Fe $\left.\left\{\mathrm{H}_{2} \mathbf{B}(\mathbf{p z})(\mathrm{pypz})\right\}_{2}\right]$ (1-I): This form can be obtained by heating 1-II at $\sim 178^{\circ} \mathrm{C}$ in an inert gas atmosphere as dark red powder.

Elemental analysis calculated for $\mathrm{C}_{22} \mathrm{H}_{22} \mathrm{~B}_{2} \mathrm{FeN}_{10}$ : C 52.43, H 4.4, N $27.79 \%$, found: C 52.03, H 4.38, N $27.39 \%$.

HRESI-MS(+)(CH3CN+MeOH): $m / z(\%)=\left[\mathrm{M}-\text { Ligand }+\mathrm{CH}_{3} \mathrm{CN}\right]^{+}$calcd. 321.07169, found 321.07121 (26); [M] $]^{+}$calcd. 504.15590, found $504.15557(71) ;[\mathrm{M}+\mathrm{H}]^{+}$ calcd. 505.16372, found 505.16288 (41).

IR (ATR): $v / \mathrm{cm}^{-1}=3126,3116,3101,3085,3069,3060(\mathrm{w}, v[=\mathrm{C}-\mathrm{H}]), 2401,2376$, 2356, 2340, $2327\left(\mathrm{~m}, v_{\text {asym. }}\left[-\mathrm{BH}_{2}\right]\right), 2278,2264\left(\mathrm{~m}, v_{\text {sym. }}\left[-\mathrm{BH}_{2}\right]\right), 1603(\mathrm{~m}), 1566(\mathrm{w}), 1525(\mathrm{w})$, 1505 (w), 1484 (w), 1452 (m), 1431 (m), 1399 (m), 1376 (w), 1350 (m), 1290 (m), 1245 (w), 1186 (m), 1147 (s), 1133 (w), 1119 (w), 1092 (m), 1972 (w), 1053 (s), 1009 (w), 976 (w), 962 (w), 945 (w), 921 (w), 878 (m), 843 (w), 790 (w), 755 (s), 725 (m), $710(w), 692(\mathrm{~m}), 666(\mathrm{~m})$, $637(\mathrm{~m}), 625(\mathrm{~m}), 610(\mathrm{w}), 514(\mathrm{w}), 478(\mathrm{w}), 455(\mathrm{w}), 410(\mathrm{~m})$.

Raman (Bulk): $v / \mathrm{cm}^{-1}=3060$ (vw, $v[=\mathrm{C}-\mathrm{H}]$ ), 2403, 2337 (vw, vasym.[-BH 2$]$ ), 1605 (s), 1566 (m), 1525 (s), 1486 (m), 1432 (w), 1354 (s), 1293 (w), 1271 (w), 1197 (w), 1093 (w), $1009(\mathrm{~s}), 963(\mathrm{~m}), 708(\mathrm{w}), 636(\mathrm{w}), 310(\mathrm{w})$. 
UV/Vis (KBr): $\lambda_{\max } / \mathrm{nm}=$ 204, 253 (br), 299 (br), $438-608$ (br).

The bulk material showed no visible color change with liquid nitrogen.

The phase purity was confirmed by comparison of the experimental XRPD pattern with that calculated from the single crystal structure of $\mathbf{2}$, where the lattice parameters were optimized by a Pawley-Fit (Figure S2).

Thin films can be prepared by physical vapour deposition under similar conditions $(p=$ $10^{-2}$ mbar, $T=160{ }^{\circ} \mathrm{C}$ and $t=4 \mathrm{~min}$ ) as previously reported. ${ }^{1-6}$

Synthesis of [Zn $\left.\left\{\mathbf{H}_{2} \mathbf{B}(\mathbf{p z})(\mathbf{p y p z})\right\}_{2}\right]$ (2): To a solution of $\mathrm{Zn}(\mathrm{OTf})_{2}(183 \mathrm{mg}, 503 \mu \mathrm{mol})$ in methanol (4 mL) was filtered off a solution of $\mathrm{K}\left[\mathrm{H}_{2} \mathrm{~B}(\mathrm{pz})(\mathrm{pypz})\right](263 \mathrm{mg}, 1.00 \mathrm{mmol})$ in methanol $(5 \mathrm{~mL})$, leading to the formation of a colorless solution. The reaction mixture was stirred at room-temperature. After a few minutes a colorless precipitate was formed. The precipitate was collected, washed with methanol $(1 \mathrm{~mL})$ and dried by suction filtration. Yield: $89 \mathrm{mg}\left(173 \mu \mathrm{mol}, 34 \%\right.$ vs. $\left.\mathrm{Zn}(\mathrm{OTf})_{2}\right)$. If the filtrate is stored at $5{ }^{\circ} \mathrm{C}$, colorless platelets-like crystals suitable for single crystal structure determination has been obtained after a few days.

Elemental analysis calculated for $\mathrm{C}_{22} \mathrm{H}_{22} \mathrm{~B}_{2} \mathrm{ZnN}_{10}$ : C 51.46, H 4.32, N $27.28 \%$, found: C 50.97, H 4.30, N $26.96 \%$.

HRESI-MS(+)(CHCl $3+\mathbf{M e O H}): m / z(\%)=[\mathrm{M}+\mathrm{H}]^{+}$calcd. 513.15792, found 513.15799 (100).

${ }^{1} \mathbf{H}$ NMR (400 MHz, CD2 $\left.\mathbf{C l}_{2}\right): \delta / \mathrm{ppm}=7.90\left(\mathrm{~d}, \mathrm{~J}=2.2 \mathrm{~Hz}, 1 \mathrm{H}\right.$, pyrazolyl $\left.-\mathrm{H}^{5}\right), 7.55(\mathrm{~m}$, $3 \mathrm{H}$, pyrazolyl- $\mathrm{H}^{5 \mathrm{~A}}$, pyridyl- $\left.\mathrm{H}^{7},-\mathrm{H}^{10}\right), 7.16\left(\mathrm{ddd}, \mathrm{J}=5.0 \mathrm{~Hz}, 1.7 \mathrm{~Hz}, 1.0 \mathrm{~Hz}, 1 \mathrm{H}\right.$, pyridyl- $\mathrm{H}^{8}$ or $\left.\mathrm{H}^{9}\right), 7.11\left(\mathrm{dd}, \mathrm{J}=2.0 \mathrm{~Hz}, 0.7 \mathrm{~Hz}, 1 \mathrm{H}\right.$, pyrazolyl-H $\left.{ }^{3 \mathrm{~A}}\right), 6.84(\mathrm{ddd}, \mathrm{J}=7.2 \mathrm{~Hz}, 5.0 \mathrm{~Hz}, 1.5 \mathrm{~Hz}$, $1 \mathrm{H}$, pyridyl- $\mathrm{H}^{8}$ or $\left.-\mathrm{H}^{9}\right), 6.79\left(\mathrm{~d}, \mathrm{~J}=2.2 \mathrm{~Hz}, 1 \mathrm{H}\right.$, pyrazolyl- $\left.\mathrm{H}^{4}\right), 5.92(\mathrm{t}, \mathrm{J}=2.1 \mathrm{~Hz}, 1 \mathrm{H}$, pyrazolyl$\mathrm{H}^{4 \mathrm{~A}}$ ), 3.76 (br. d, J = $124.3 \mathrm{~Hz}, 2 \mathrm{H}, \mathrm{B}-\mathrm{H}$ ).

${ }^{13} \mathbf{C}\left\{{ }^{1} \mathbf{H}\right\}$ NMR (100 MHz, $\left.\mathbf{C D}_{2} \mathbf{C l}_{2}\right): \delta / p p m=148.76\left(\mathrm{C}_{\mathrm{q}}\right.$, pyrazolyl-C $\left.\mathrm{C}^{3}\right), 148.51\left(\mathrm{C}_{\mathrm{q}}\right.$, pyridyl- $\left.\mathrm{C}^{6}\right), 147.09\left(\mathrm{CH}\right.$, pyridyl- $\mathrm{C}^{8}$ or $\left.-\mathrm{C}^{9}\right), 140.33\left(\mathrm{CH}\right.$, pyrazolyl- $\left.\mathrm{C}^{3 \mathrm{~A}}\right), 138.25(\mathrm{CH}$, pyridyl$\mathrm{C}^{7}$ or $\left.-\mathrm{C}^{10}\right), 138.02\left(\mathrm{CH}\right.$, pyrazolyl- $\left.\mathrm{C}^{5}\right), 136.39\left(\mathrm{CH}\right.$, pyrazolyl- $\left.\mathrm{C}^{5 \mathrm{~A}}\right), 123.41\left(\mathrm{CH}\right.$, pyrazolyl-C ${ }^{8}$ or $\left.-\mathrm{C}^{9}\right), 119.90\left(\mathrm{CH}\right.$, pyridyl- $\mathrm{C}^{7}$ or $\left.-\mathrm{C}^{10}\right), 103.96\left(\mathrm{CH}\right.$, pyrazolyl- $\left.\mathrm{C}^{4 \mathrm{~A}}\right), 101.93(\mathrm{CH}$, pyrazolyl$\left.\mathrm{C}^{4}\right)$.

${ }^{11} \mathrm{~B}$ NMR (128 MHz, CD2Cl2): $\delta / \mathrm{ppm}=-8.41$ (br. s, 1B).

IR (ATR): $v / \mathrm{cm}^{-1}=3140,3116,3081,3056,3015(\mathrm{w}, v[=\mathrm{C}-\mathrm{H}]), 2448,2407,2360$, 2344, $2332\left(\mathrm{~m}, v_{\text {asym. }}\left[-\mathrm{BH}_{2}\right]\right), 2280,2268\left(\mathrm{w}, v_{\text {sym. }}\left[-\mathrm{BH}_{2}\right]\right), 1719(\mathrm{w}), 1605(\mathrm{~m}), 1570(\mathrm{~m}), 1525$ (w), 1507 (w), 1488 (m), 1456 (w), 1433 (m), 1399 (m), 1378 (m), 1354 (m), 1294 (m), 1247 (w), 1192 (m), 1178 (m), 1149 (s), 1135 (m), 1092 (m), 1076 (m), 1053 (s), 1007 (m), 999(m), 
978 (m), 964 (w), $921(\mathrm{w}), 894(\mathrm{w}), 878(\mathrm{~m}), 845$ (w), $796(\mathrm{w}), 774(\mathrm{~m}), 759(\mathrm{~s}), 725$ (s), 706 (w), $692(\mathrm{~m}), 668(\mathrm{~m}), 641(\mathrm{~m}), 629(\mathrm{~m}), 517(\mathrm{w}), 478(\mathrm{w}), 414(\mathrm{w}), 408(\mathrm{~m})$.

Raman (Bulk): $v / \mathrm{cm}^{-1}=3151,3142,3130,3117,3107,3078,3059$ (w, $v[=\mathrm{C}-\mathrm{H}]$ ), 2449, 2411, 2372, 2361, 2345, 2334 (m, vasym. [-BH 2$]), 2293,2281,2270$ (w, v sym. $\left.\left[-\mathrm{BH}_{2}\right]\right), 1605$ (s), 1570 (m), 1527 (s), 1491 (m), 1458 (w), 1427 (w), 1404 (w), 1379 (w), 1358 (s), 1296 (w), 1277 (w), 1248 (w), 1205 (w), 1178 (w), 1153 (w), 1093 (w), 1076 (w), 1049 (w), 1009 (m), $964(\mathrm{~m}), 924(\mathrm{w}), 795$ (w), 708 (w), 634 (w), $615(\mathrm{w}), 511(\mathrm{w}), 478(\mathrm{w}), 384(\mathrm{w})$.

UV/Vis (KBr): $\lambda_{\max } / \mathrm{nm}=209,255,297,610-672$ (br).

To further characterize compound $\mathbf{2}$ a single crystal structure determination has been performed. The experimental XRPD pattern as well as the calculated pattern obtained from the single crystal structure proves that a pure phase has been obtained (Figure S3).

Thin films can be prepared by physical vapour deposition under similar conditions ( $p=$ $10^{-2}$ mbar, $T=180^{\circ} \mathrm{C}$ and $t=2 \mathrm{~min}$ ) as previously reported. ${ }^{1-6}$

Synthesis of [Co\{ $\left.\left.\mathbf{H}_{2} \mathbf{B}(\mathbf{p z})(\mathbf{p y p z})\right\}_{2}\right](\mathbf{3})$ : To a solution of $\mathrm{Co}(\mathrm{OTf})_{2}(179 \mathrm{mg}, 500 \mu \mathrm{mol})$ in methanol $(20 \mathrm{~mL})$ a solution of $\mathrm{K}\left[\mathrm{H}_{2} \mathrm{~B}(\mathrm{pz})(\mathrm{pypz})\right](263 \mathrm{mg}, 1.00 \mathrm{mmol})$ in methanol $(5 \mathrm{~mL})$ was added dropwise, leading to the formation of a pink colored solution. The solution was stirred overnight under $\mathrm{N}_{2}$. Afterwards a pink colored precipitate was formed, which was filtered off, washed with methanol $(5 \mathrm{~mL})$ and dried under reduced pressure $(1 \mathrm{~h})$. Yield: $56 \mathrm{mg}$ $\left(110 \mu \mathrm{mol}, 22 \%\right.$ vs. $\left.\mathrm{Co}(\mathrm{OTf})_{2}\right)$. In this case a microcrystalline powder is obtained that is isotypic to 2 . If the filtrate is stored at $5{ }^{\circ} \mathrm{C}$, orange block-like crystals of a methanol solvate (3$\mathrm{MeOH}$ ) suitable for single crystal structure determination has been obtained within a few days.

Elemental analysis calculated for $\mathrm{C}_{22} \mathrm{H}_{22} \mathrm{~B}_{2} \mathrm{CoN}_{10}$ : C 52.11, H 4.37, N $27.62 \%$, found: C 51.84, H 4.41, N $27.52 \%$.

HRESI-MS(+) $\left(\mathbf{C H}_{3} \mathbf{C N}+\mathbf{M e O H}\right): m / z(\%)=[\mathrm{M}]^{+}$calcd. 507.15415 , found 507.15431 (100).

IR (ATR): $v / \mathrm{cm}^{-1}=3138,3113,3081,3060(\mathrm{w}, v[=\mathrm{C}-\mathrm{H}]), 2405,2358,2344,2329(\mathrm{~m}$,

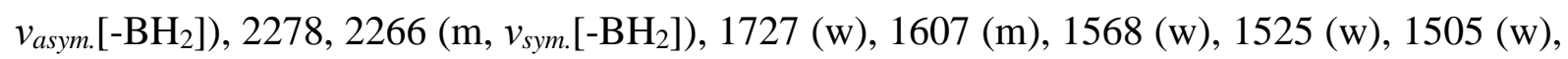
1486 (m), 1454 (w), 1435 (m), 1401 (m), 1378 (w), 1354 (w), 1292 (m), 1271 (w), 1247 (w), 1192 (m), 1180 (m), 1149 (s), 1135 (m), 1092 (m), 1074 (m), 1053 (s), 1011 (w), 996 (w), 976 (w), $964(\mathrm{w}), 921(\mathrm{w}), 894(\mathrm{w}), 880(\mathrm{~m}), 864(\mathrm{w}), 845(\mathrm{w}), 794(\mathrm{w}), 772(\mathrm{w}), 759(\mathrm{~s}), 733(\mathrm{w})$, 725 (m), 706 (w), 692 (m), $668(\mathrm{~m}), 637(\mathrm{~m}), 627(\mathrm{~m}), 612(\mathrm{w}), 517(\mathrm{w}), 480(\mathrm{w}), 419(\mathrm{w}), 410$ (w). 
Raman (Bulk): $v / \mathrm{cm}^{-1}=3241,3130,3114,3060(\mathrm{~m}, v[=\mathrm{C}-\mathrm{H}]), 2406\left(\mathrm{~m}, v_{\text {asym. }}\left[-\mathrm{BH}_{2}\right]\right)$, 2269 (vw, v sym. [-BH 2$]), 1605$ (s), 1566 (m), 1524 (s), 1486 (m), 1354 (s), 1292 (w), 1273 (w), $1195(w), 1093(w), 1076(w), 1052(w), 1009(m), 963(w), 922(w)$.

UV/Vis (KBr): $\lambda_{\max } / \mathrm{nm}=213,258,300,465-608$ (br).

To further characterize compound $\mathbf{3} \cdot 2.5$ methanol a single crystal structure determination has been performed. The experimental XRPD pattern as well as the simulated pattern calculated from the single crystal structural data of $\mathbf{2}$ proves that a pure phase has been obtained (Figure S4).

Thin films can be prepared by physical vapour deposition under similar conditions $(p=$ $10^{-2}$ mbar, $T=180{ }^{\circ} \mathrm{C}$ and $t=2 \mathrm{~min}$ ) as previously reported. ${ }^{1-6}$

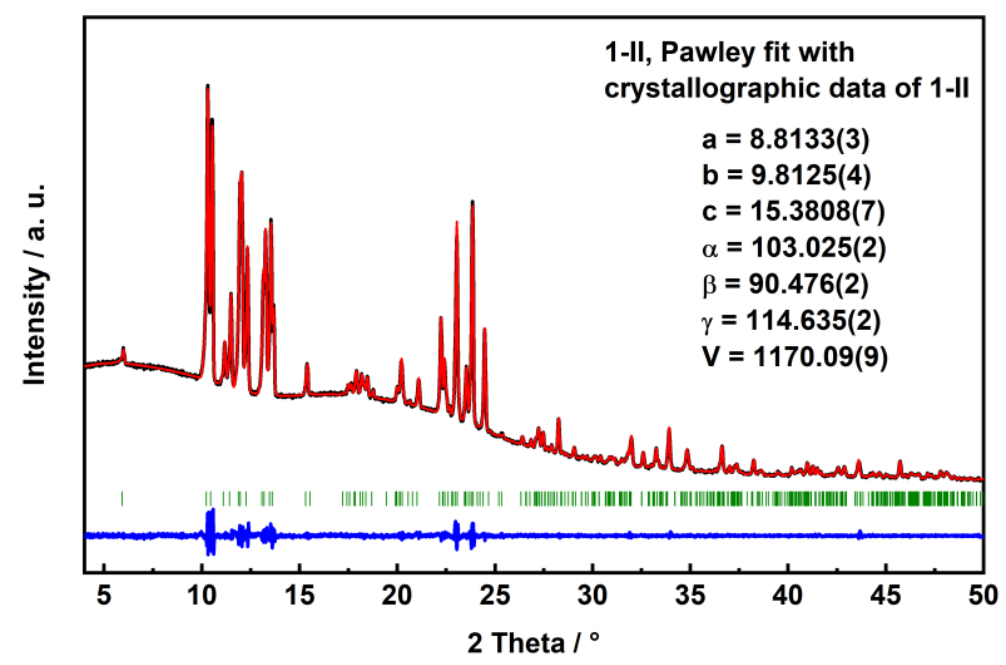

Figure S1. Experimental powder pattern (black), Pawley fit (red), hkl ticks (green) and difference plot (blue) of 1-II (synthesis product) using the crystallographic data of compound 1-II at $200 \mathrm{~K}$. 


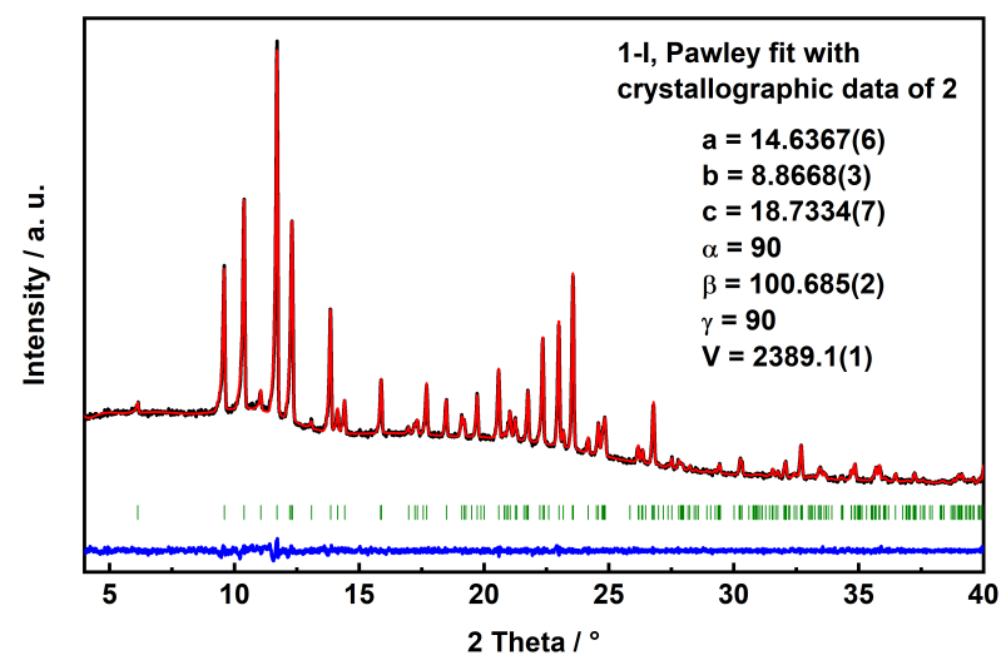

Figure S2. Experimental powder pattern (black), Pawley fit (red), hkl ticks (green) and difference plot (blue) of 1-I (prepared by a TG measurement) using the crystallographic data of compound 2.

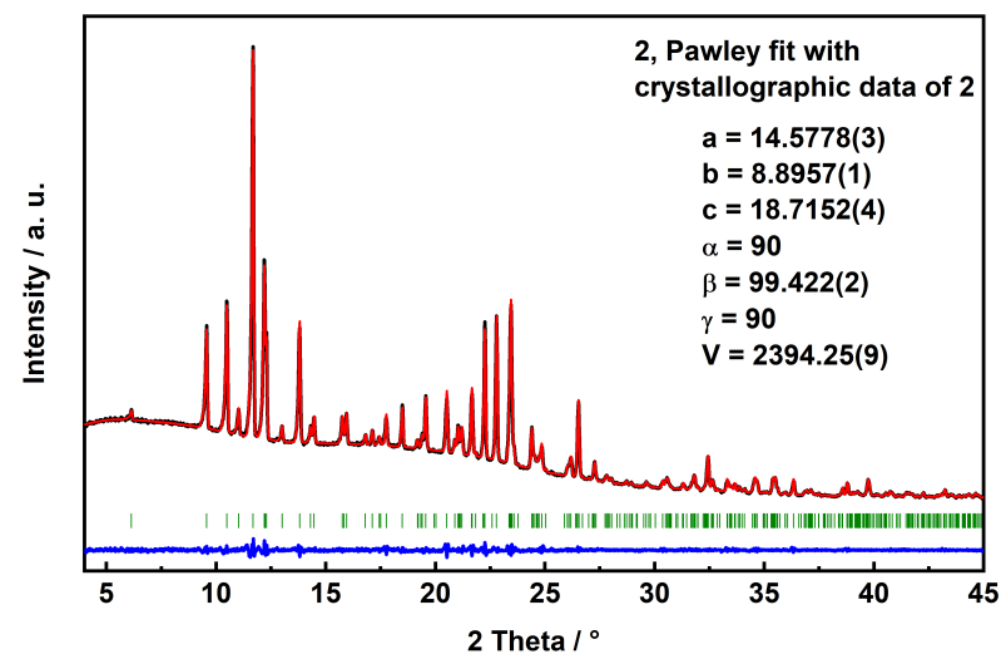

Figure S3. Experimental powder pattern (black), Pawley fit (red), hkl ticks (green) and difference plot (blue) of 2 (synthesis product) using the crystallographic data of compound $\mathbf{2}$. 


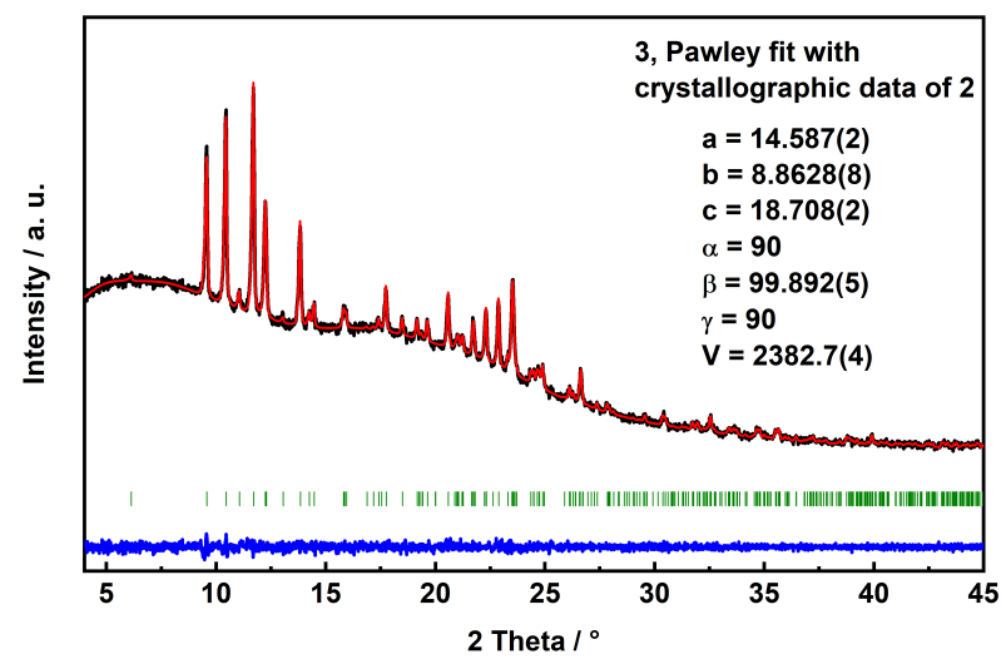

Figure S4. Experimental powder pattern (black), Pawley fit (red), hkl ticks (green) and difference plot (blue) of $\mathbf{3}$ using the crystallographic data of compound 2. 
Table S1. Crystal data and details of the structure refinement for 1-II, 2 and 3-MeOH at $200 \mathrm{~K}$.

\begin{tabular}{|c|c|c|c|}
\hline Empirical formula & $\mathrm{C}_{22} \mathrm{H}_{22} \mathrm{~B}_{2} \mathrm{~N}_{10} \mathrm{Fe}$ & $\mathrm{C}_{22} \mathrm{H}_{22} \mathrm{~B}_{2} \mathrm{~N}_{10} \mathrm{Zn}$ & $\mathrm{C}_{24.5} \mathrm{H}_{32.5} \mathrm{~B}_{2} \mathrm{~N}_{10} \mathrm{O}_{2.5} \mathrm{Co}$ \\
\hline Formula weight & 503.96 & 513.48 & 587.65 \\
\hline Crystal size, $\mathrm{mm}^{3}$ & $0.08 \times 0.15 \times 0.22$ & $0.02 \times 0.08 \times 0.12$ & $0.08 \times 0.16 \times 0.22$ \\
\hline Crystal system & Triclinic & Monoclinic & Monoclinic \\
\hline Space group & $P-1$ & $P 2{ }_{1} / c$ & $C 2 / c$ \\
\hline Temperature, $\mathrm{K}$ & $200(2)$ & $200(2)$ & $200(2)$ \\
\hline $\mathrm{a}, \AA$ & $8.7257(3)$ & $14.4740(5)$ & $38.4739(12)$ \\
\hline $\mathrm{b}, \AA$ & $9.7725(4)$ & $8.8342(2)$ & $11.3079(2)$ \\
\hline c, $\AA$ & $15.2694(6)$ & $18.6381(6)$ & $13.7515(5)$ \\
\hline$\alpha,^{\circ}$ & $102.809(3)$ & 90 & 90 \\
\hline$\beta,{ }^{\circ}$ & $90.625(3)$ & $99.507(3)$ & $107.159(2)$ \\
\hline$\gamma,{ }^{\circ}$ & $114.620(3)$ & 90 & 90 \\
\hline $\mathrm{V}, \AA^{3}$ & $1146.37(8)$ & $2350.45(12)$ & $5716.4(3)$ \\
\hline $\mathrm{Z}$ & 2 & 4 & 8 \\
\hline Density (calculated), $\mathrm{Mg} / \mathrm{m}^{3}$ & 1.460 & 1.451 & 1.366 \\
\hline Parameters & 332 & 332 & 384 \\
\hline Absorption coefficient, $\mathrm{mm}^{-1}$ & 0.692 & 1.078 & 0.644 \\
\hline $\mathrm{F}(000)$ & 520 & 1056 & 2452 \\
\hline Theta range for data collection, ${ }^{\circ}$ & 1.377 to 28.000 & 1.426 to 26.005 & 1.884 to 26.004 \\
\hline Reflections collected & 18459 & 15087 & 20777 \\
\hline Independent reflections & 5538 & 4590 & 5589 \\
\hline $\mathrm{R}(\mathrm{int})$ & 0.0551 & 0.0330 & 0.0379 \\
\hline Observed reflections $(\mathrm{I}>2 \sigma(\mathrm{I}))$ & 4855 & 4041 & 4798 \\
\hline $\mathrm{R} 1(\mathrm{I}>2 \sigma(\mathrm{I}))$ & 0.0349 & 0.0367 & 0.0458 \\
\hline wR2 (all data) & 0.0902 & 0.0960 & 0.1229 \\
\hline Goodness-of-fit on $\mathrm{F}^{2}$ & 1.050 & 1.048 & 1.055 \\
\hline Largest diff, peak and hole, e. $\AA^{-3}$ & 0.350 and -0.372 & 0.941 and -0.664 & 0.428 and -0.380 \\
\hline
\end{tabular}


Table S2. Crystal data and structure refinement for 1-II at 300, 393, $423 \mathrm{~K}$ and $300 \mathrm{~K}$ after cooling from $393 \mathrm{~K}$.

\begin{tabular}{|c|c|c|c|c|}
\hline Temperature, $\mathrm{K}$ & $303(2)$ & 393(2) & 423(2) & $300(2)$ \\
\hline Empirical formula & $\mathrm{C}_{22} \mathrm{H}_{22} \mathrm{~B}_{2} \mathrm{~N}_{10} \mathrm{Fe}$ & $\mathrm{C}_{22} \mathrm{H}_{22} \mathrm{~B}_{2} \mathrm{~N}_{10} \mathrm{Fe}$ & $\mathrm{C}_{22} \mathrm{H}_{22} \mathrm{~B}_{2} \mathrm{~N}_{10} \mathrm{Fe}$ & $\mathrm{C}_{22} \mathrm{H}_{22} \mathrm{~B}_{2} \mathrm{~N}_{10} \mathrm{Fe}$ \\
\hline Formula weight & 503.96 & 503.96 & 503.96 & 503.96 \\
\hline Crystal size, $\mathrm{mm}^{3}$ & $\begin{array}{l}0.02 \times 0.06 \times \\
0.10\end{array}$ & $\begin{array}{l}0.025 \times 0.05 \times \\
0.16\end{array}$ & $\begin{array}{l}0.025 \times 0.05 \times \\
0.16\end{array}$ & $\begin{array}{l}0.025 \times 0.05 \times \\
0.16\end{array}$ \\
\hline Crystal system & Triclinic & Triclinic & Triclinic & Triclinic \\
\hline Space group & $P-1$ & $P-1$ & $P-1$ & $P-1$ \\
\hline $\mathrm{a}, \AA$ & $8.7837(2)$ & $8.8804(5)$ & $8.942(4)$ & $8.7696(2)$ \\
\hline $\mathrm{b}, \AA$ & $9.8128(2)$ & $9.9640(5)$ & $10.129(5)$ & $9.8030(3)$ \\
\hline $\mathrm{c}, \AA$ & $15.3580(3)$ & $15.4684(8)$ & $15.385(12)$ & $15.3446(4)$ \\
\hline$\alpha,{ }^{\circ}$ & $102.8715(7)$ & $103.0019(18)$ & $103.56(2)$ & $102.8462(10)$ \\
\hline$\beta,{ }^{\circ}$ & $90.5821(7)$ & $90.3564(18)$ & $89.31(2)$ & $90.6207(9)$ \\
\hline$\gamma,{ }^{\circ}$ & $114.5767(6)$ & $114.6827(18)$ & $114.952(11)$ & $114.5326(9)$ \\
\hline $\mathrm{V}, \AA^{3}$ & $1165.65(4)$ & $1203.99(11)$ & $1222.1(12)$ & $1162.09(5)$ \\
\hline $\mathrm{Z}$ & 2 & 2 & 2 & 2 \\
\hline $\begin{array}{l}\text { Density (calculated), } \\
\mathrm{Mg} / \mathrm{m}^{3}\end{array}$ & 1.436 & 1.390 & 1.369 & 1.440 \\
\hline Parameters & 332 & 332 & 332 & 332 \\
\hline $\begin{array}{l}\text { Absorption coefficient, } \\
\mathrm{mm}^{-1}\end{array}$ & 0.680 & 0.659 & 0.649 & 0.683 \\
\hline $\mathrm{F}(000)$ & 520 & 520 & 520 & 520 \\
\hline $\begin{array}{l}\text { Theta range for data } \\
\text { collection, }\end{array}$ & 2.357 to 28.283 & 2.324 to 28.277 & 2.292 to 25.023 & 2.358 to 28.276 \\
\hline Reflections collected & 31536 & 44839 & 2697 & 35741 \\
\hline Independent reflections & 5777 & 5979 & 2138 & 5765 \\
\hline $\mathrm{R}$ (int) & 0.0400 & 0.0351 & 0.0451 & 0.0397 \\
\hline $\begin{array}{l}\text { Observed reflections } \\
(\mathrm{I}>2 \sigma(\mathrm{I}))\end{array}$ & 4619 & 5162 & 1410 & 4829 \\
\hline $\mathrm{R} 1(\mathrm{I}>2 \sigma(\mathrm{I}))$ & 0.0337 & 0.0477 & 0.0727 & 0.0320 \\
\hline wR2 (all data) & 0.0801 & 0.1264 & 0.1869 & 0.0756 \\
\hline Goodness-of-fit on $\mathrm{F}^{2}$ & 1.052 & 1.062 & 1.042 & 1.052 \\
\hline $\begin{array}{l}\text { Largest diff, peak and } \\
\text { hole, e. } \AA^{-3}\end{array}$ & 0.312 and -0.339 & 0.412 and -0.258 & 0.333 and -0.323 & 0.315 and -0.302 \\
\hline
\end{tabular}




\section{Thermoanalytic investigations}

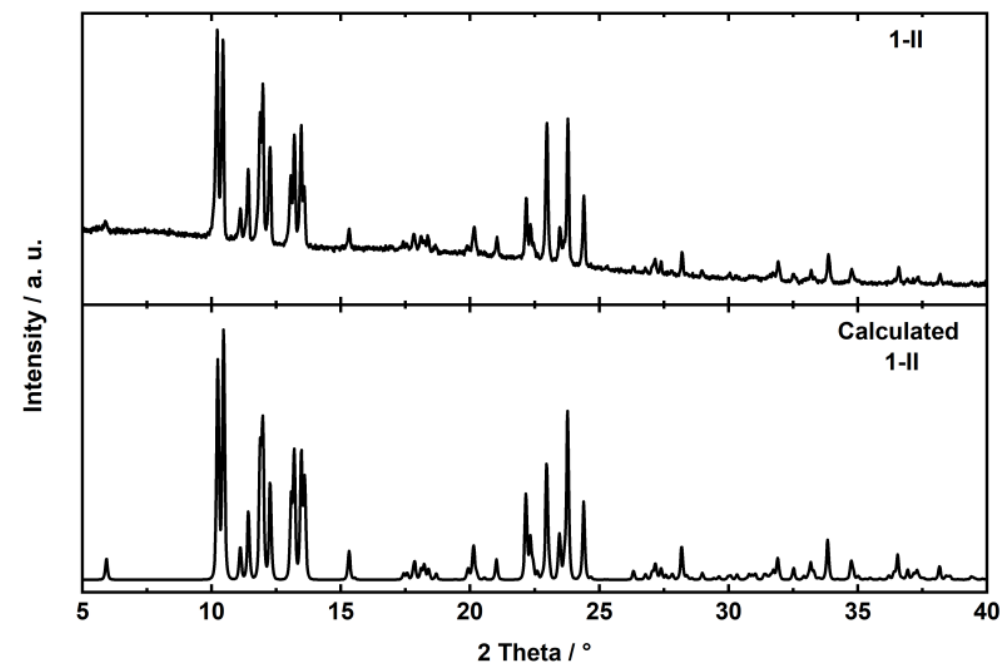

Figure S5. Experimental XRPD pattern of the residue obtained by heating to $155^{\circ} \mathrm{C}$ and cooling of 1-II (top) and calculated pattern of 1-II (bottom).

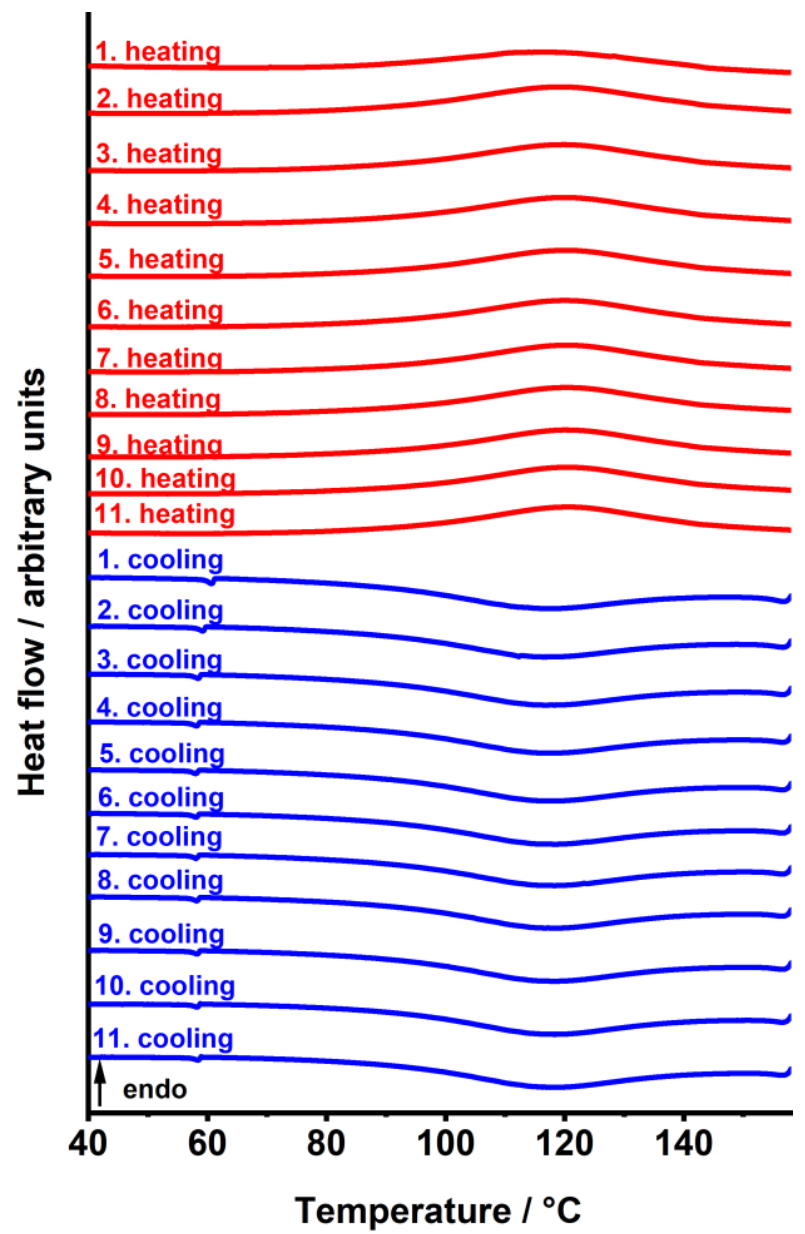

Figure S6. Heating and cooling cycles for $\mathbf{1}-\mathrm{II}$ at $10^{\circ} \mathrm{C} / \mathrm{min}$. 


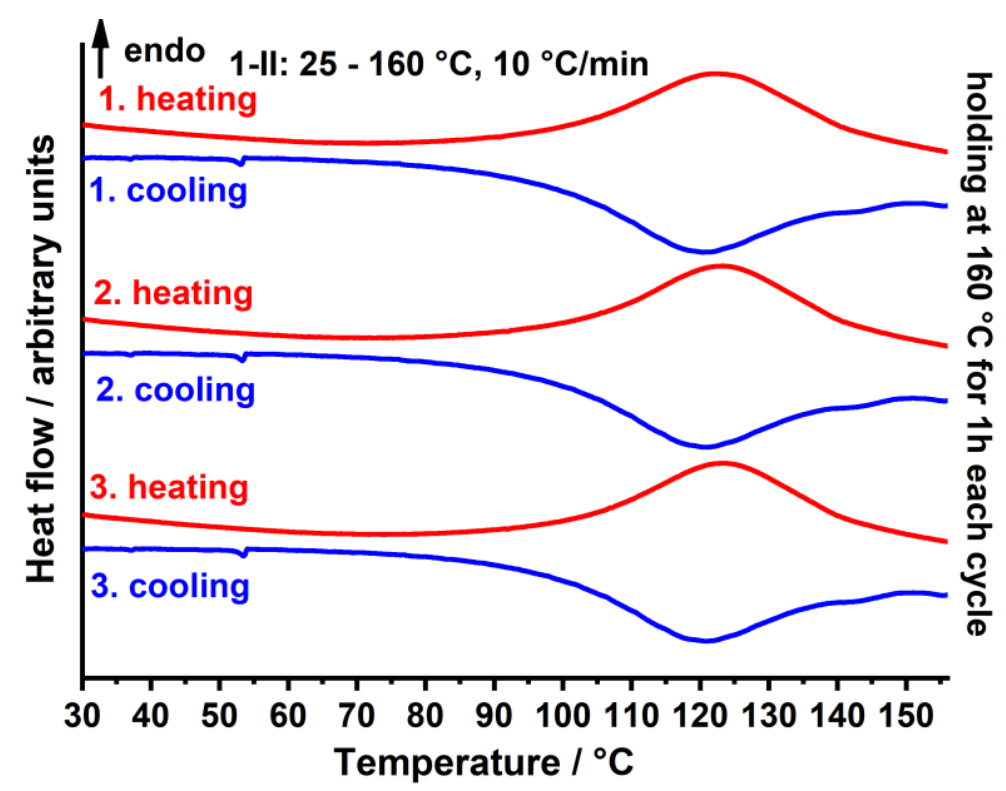

Figure S7. Heating and cooling cycles for $\mathbf{1 - I I}$ at $10{ }^{\circ} \mathrm{C} / \mathrm{min}$ between 25 and $160{ }^{\circ} \mathrm{C}$ with annealing the sample at $160{ }^{\circ} \mathrm{C}$ for $1 \mathrm{~h}$ after each cycle.

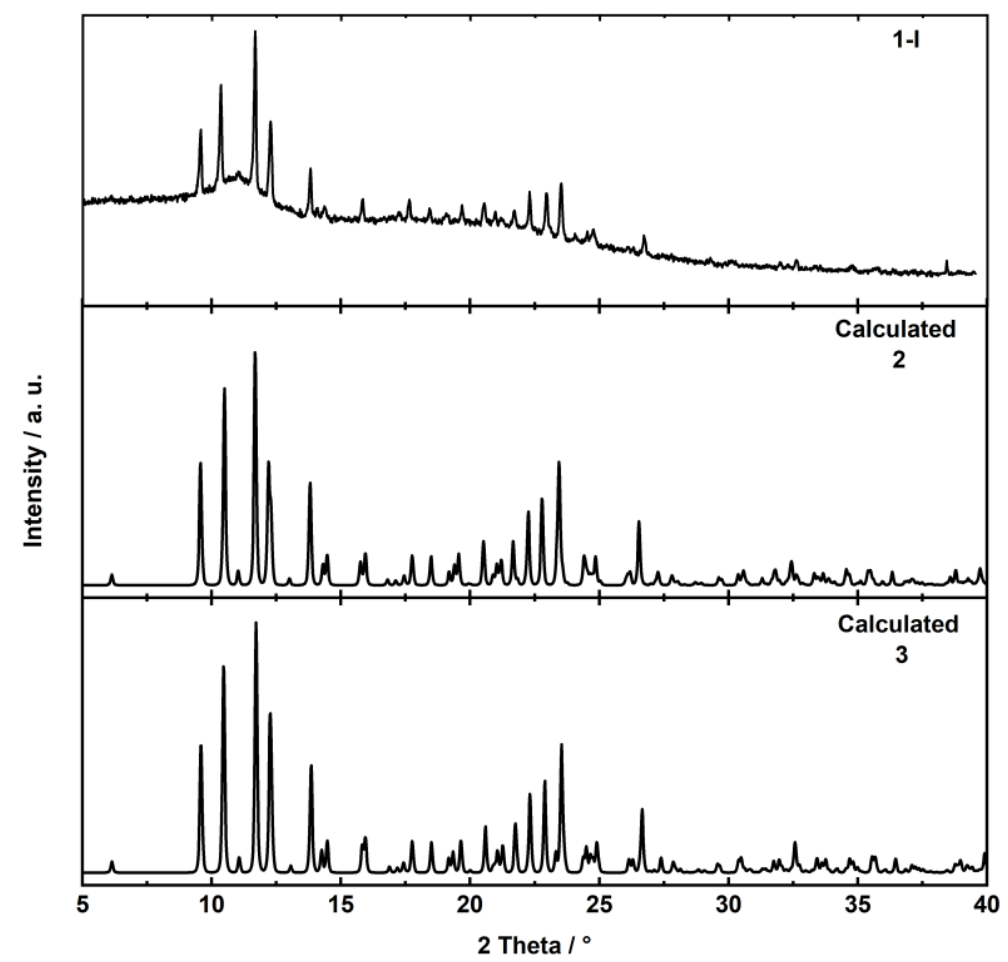

Figure S8. Experimental XRPD pattern 1-I prepared in a DSC measurement (top), calculated pattern for the Zn compound $\mathbf{2}$ (middle) and of the Co compound $\mathbf{3}$ (bottom). Powder pattern of $\mathbf{3}$ calculated starting with the crystal structure parameter of $\mathbf{2}$ and the lattice parameter for $\mathbf{3}$ obtained by a Pawley Fit. 


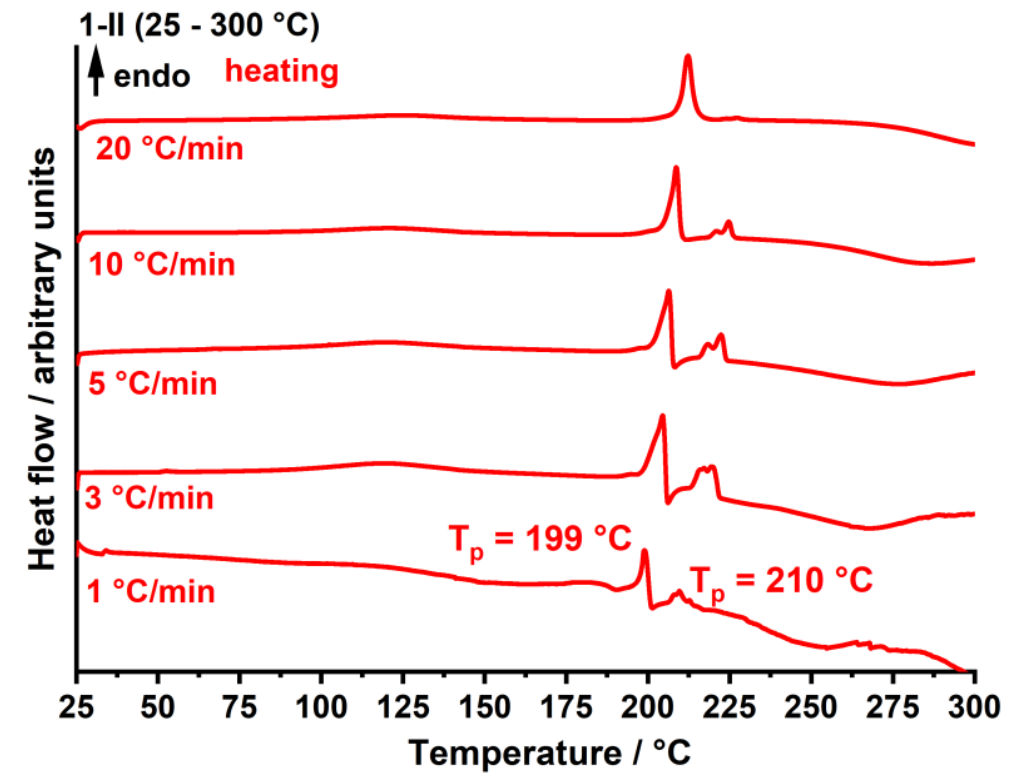

Figure S9. DSC curves of 1-II at different heating rates. 


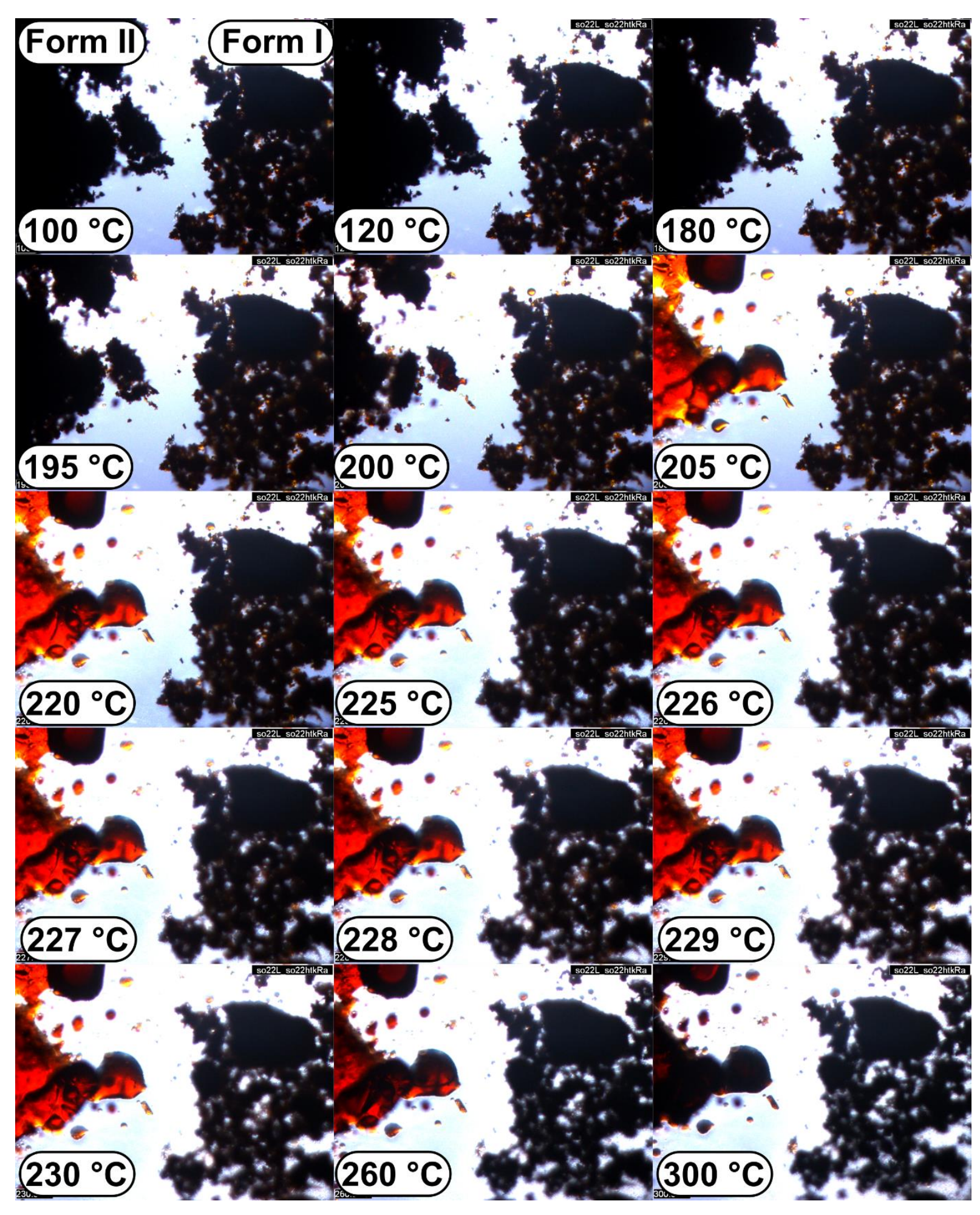

Figure S10. Microscopic images of 1-II (left) and 1-I (right) at different temperatures. 


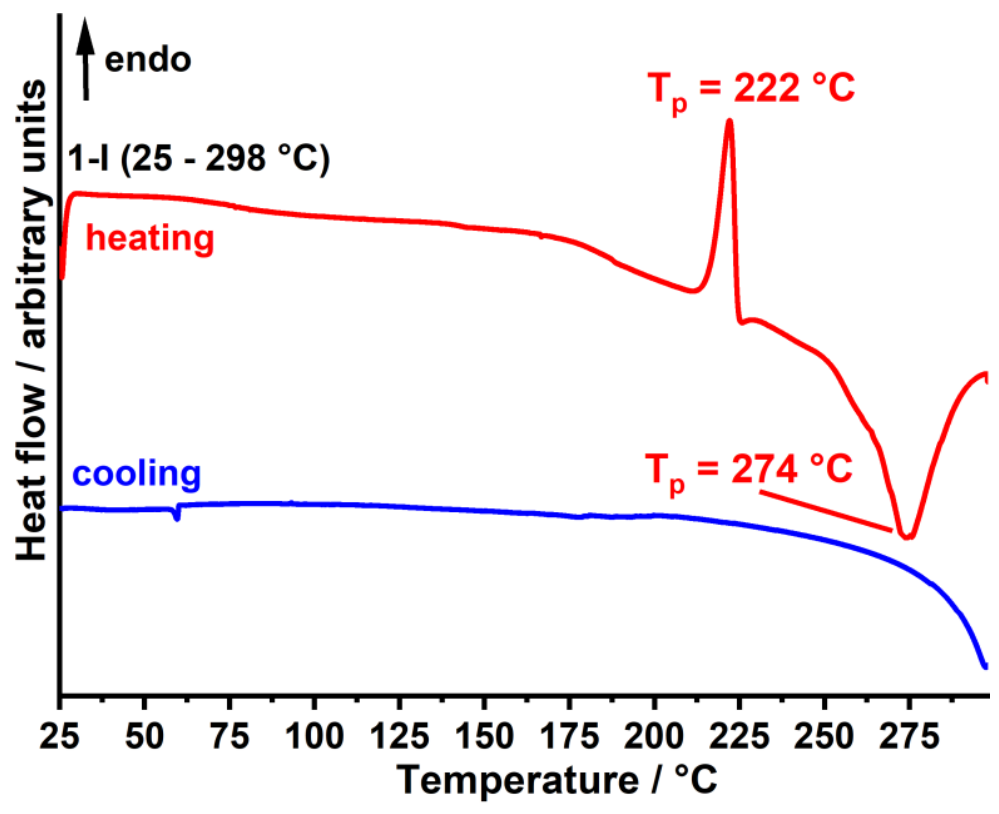

Figure S11. DSC curve of $\mathbf{1 - I}$ at $10^{\circ} \mathrm{C} / \mathrm{min}$.

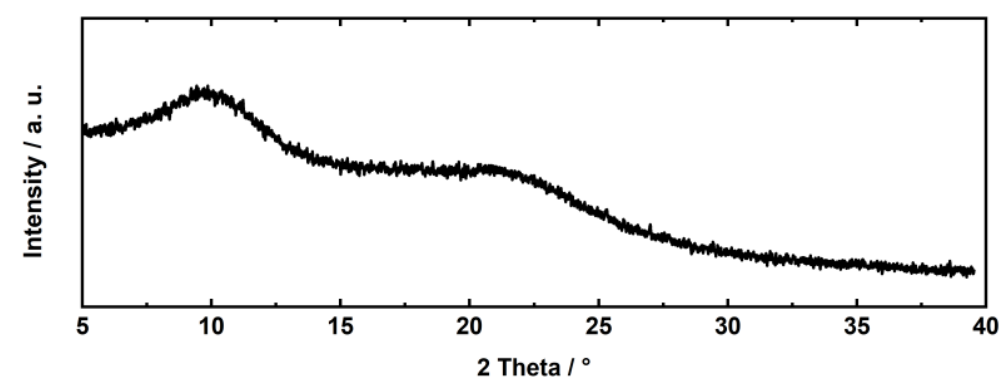

Figure S12. Experimental XRPD pattern of the residue obtained after the exothermic peak observed in an DSC measurement of 1-I.

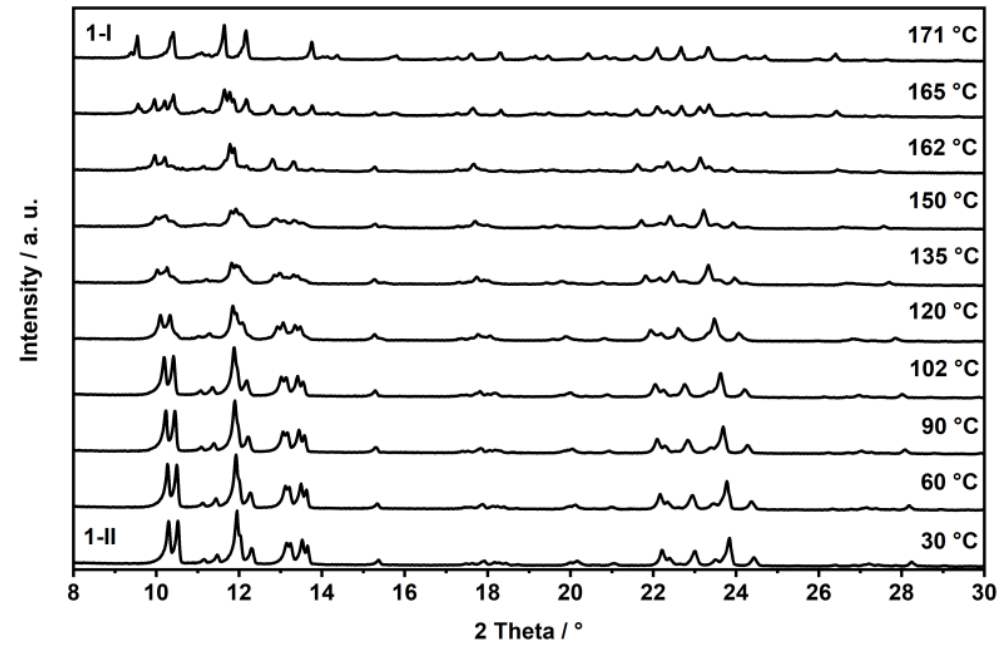

Figure S13. Selected XRPD patterns of 1-II measured at different temperatures. 


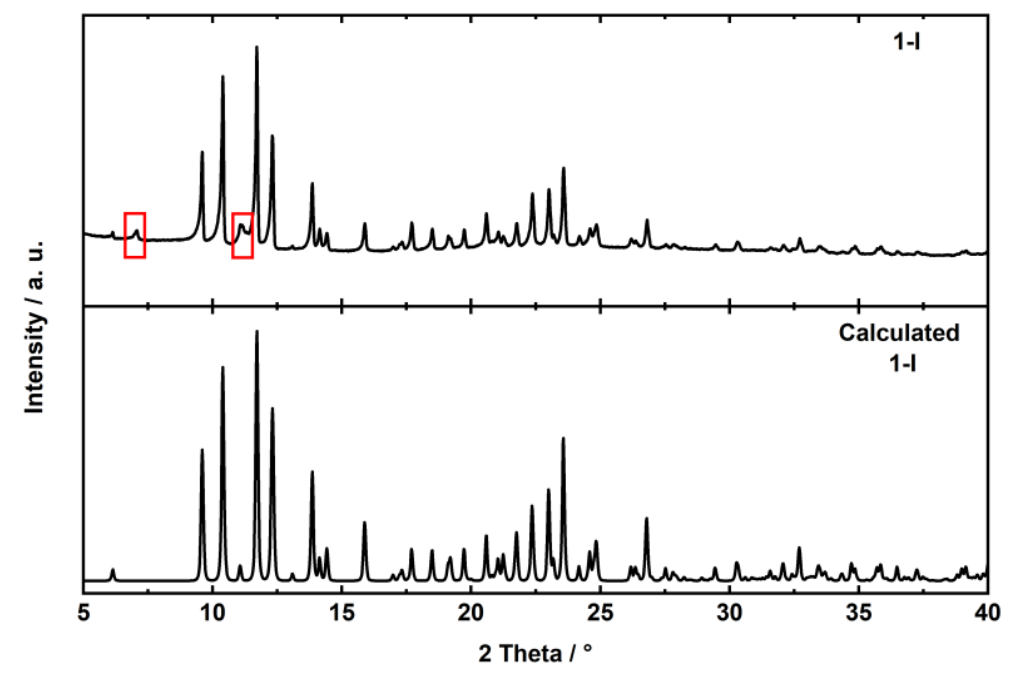

Figure S14. Experimental XRPD pattern of the residue obtained by heating of 1-II to $171{ }^{\circ} \mathrm{C}$ (top) measured at room temperature and calculated pattern of 1-I (bottom). The red boxes mark the reflections of a small contamination.

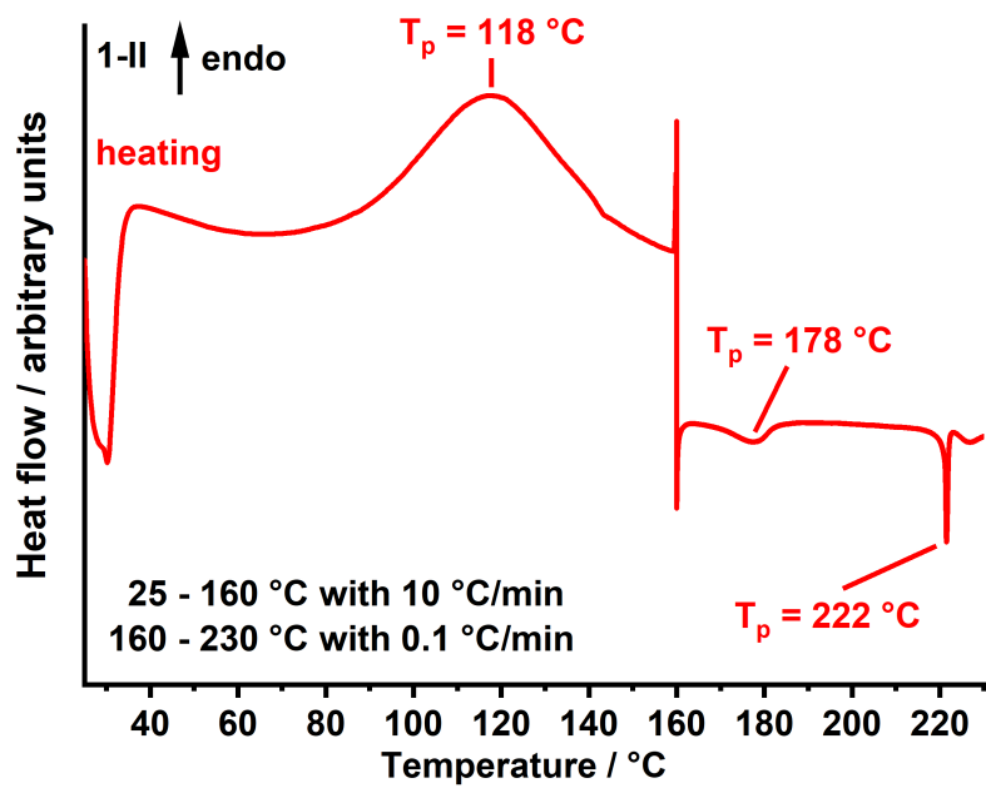

Figure S15. DSC curve of form 1-II at $10^{\circ} \mathrm{C} / \mathrm{min}$ from 25 to $160{ }^{\circ} \mathrm{C}$ and at $0.1{ }^{\circ} \mathrm{C} / \mathrm{min}$ from 160 to $230{ }^{\circ} \mathrm{C}$. The anomaly at $160^{\circ} \mathrm{C}$ originate from a change of the heating rate. 


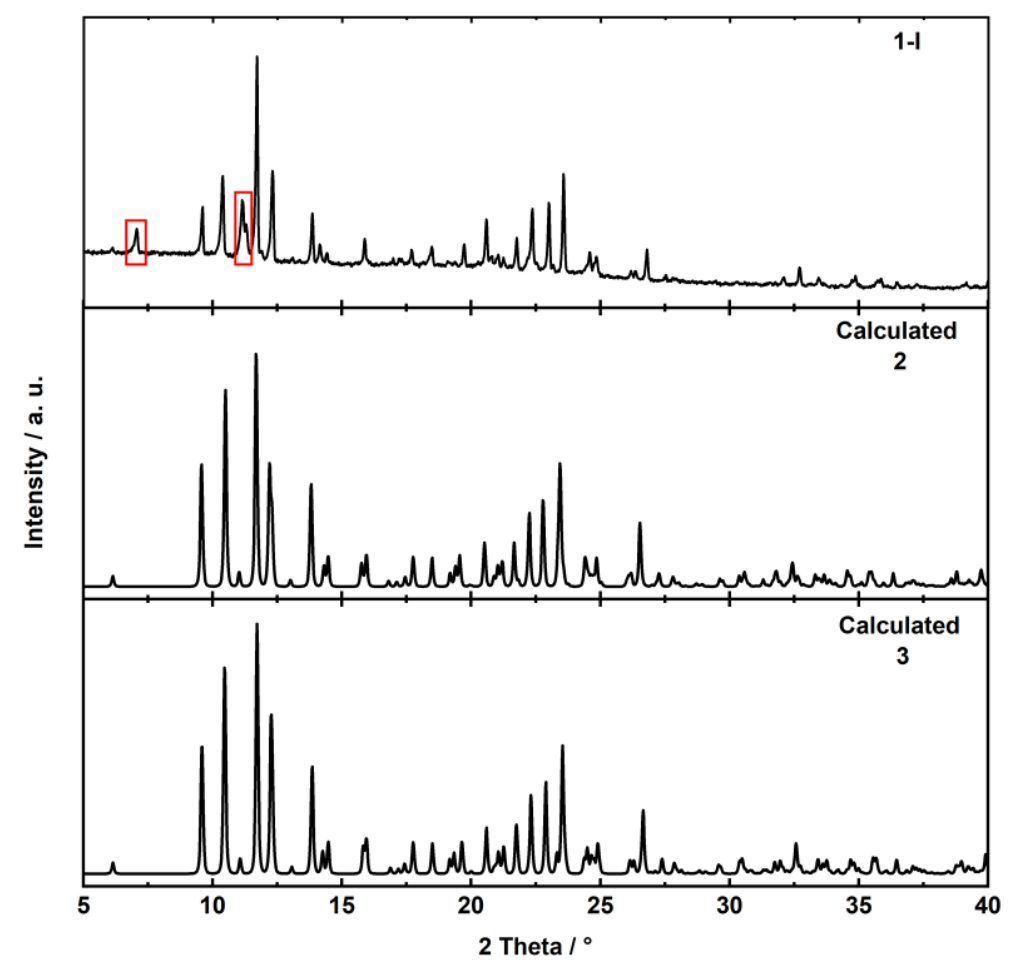

Figure S16. Experimental XRPD pattern of the residue obtained after the exothermic event at $178^{\circ} \mathrm{C}$ observed in an DSC measurement of 1-II (top), calculated pattern for the Zn compound 2 (middle) and of the Co compound $\mathbf{3}$ (bottom). Powder pattern of $\mathbf{3}$ calculated starting with the crystal structure parameter of $\mathbf{2}$ and the lattice parameter for $\mathbf{3}$ obtained by a Pawley Fit. The red boxes indicate a small contamination.

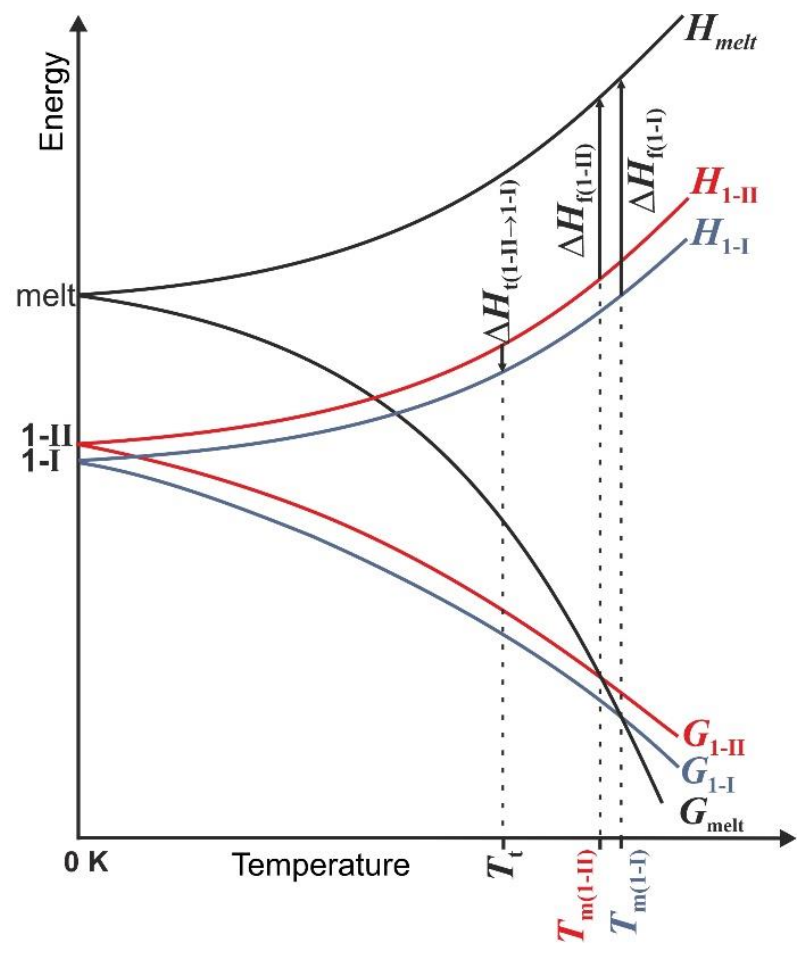

Figure S17. Semi-empirical energy-temperature diagram showing the thermodynamic relations between 1-I and 1-II. 


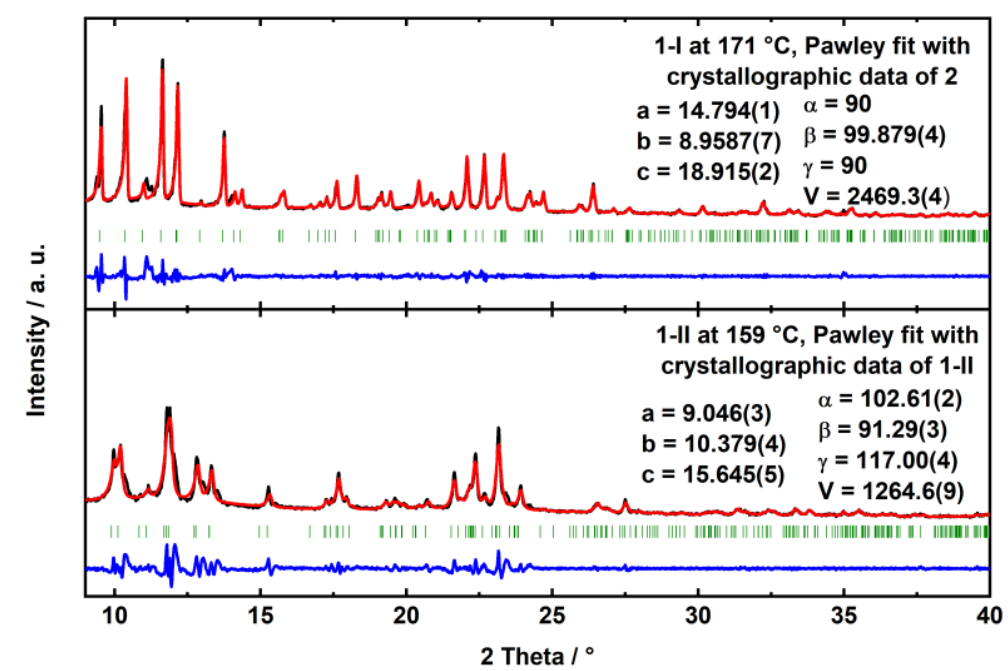

Figure S18. Pawley fits of powder patterns of 1-I measured at $171{ }^{\circ} \mathrm{C}$ (top) and of $\mathbf{1 - I I}$ measured at $159{ }^{\circ} \mathrm{C}$ (bottom).

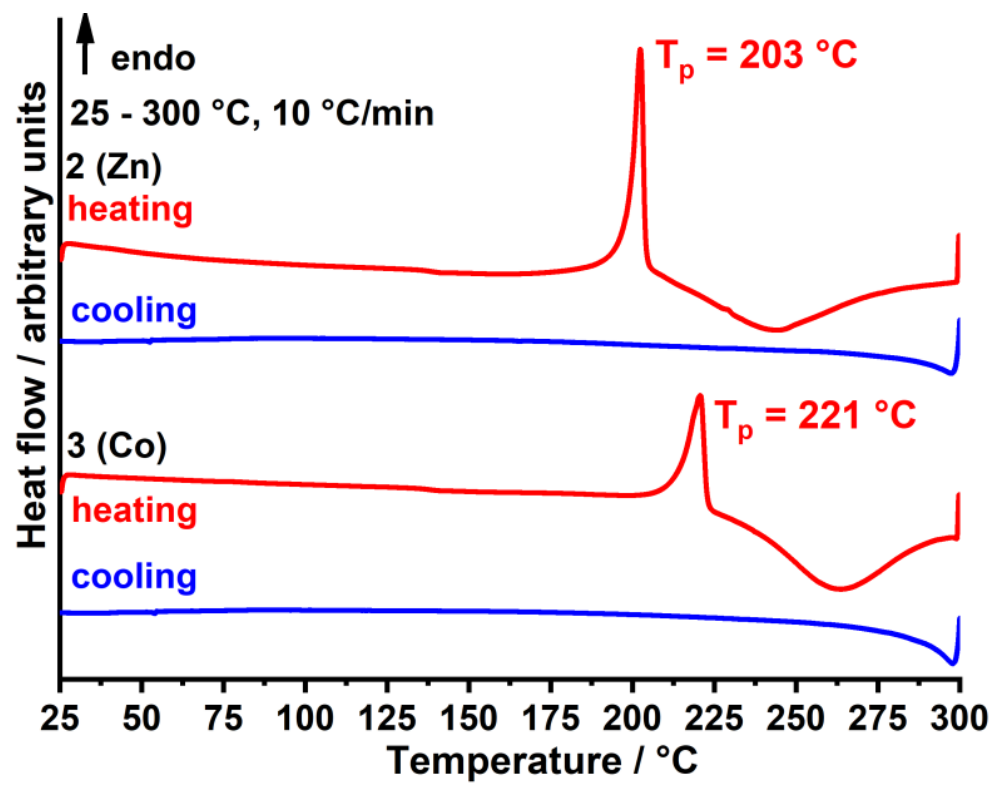

Figure S19. DSC heating and cooling curve of $\mathbf{2}$ (top) and $\mathbf{3}$ (bottom) at $10^{\circ} \mathrm{C} / \mathrm{min}$ from 25 to $300{ }^{\circ} \mathrm{C}$.

\section{Magnetic Susceptibility and Mößbauer Spectroscopic Measurements}

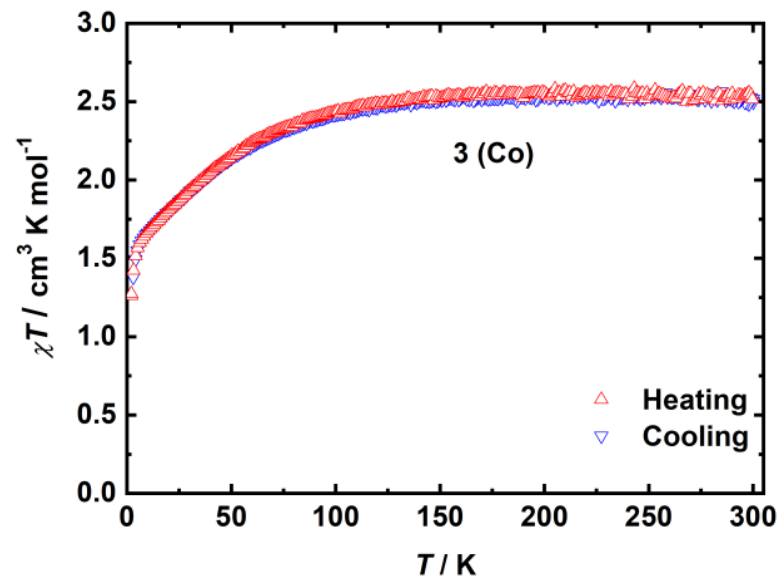


Figure S20. $\chi_{M} T$ vs $T$ curves from PPMS measurements of compound $\mathbf{3}$ between 2 and $300 \mathrm{~K}$. The curves resulting from cooling (blue symbols) and heating (red symbols) the sample almost coincide.

\section{Vibrational spectroscopy}
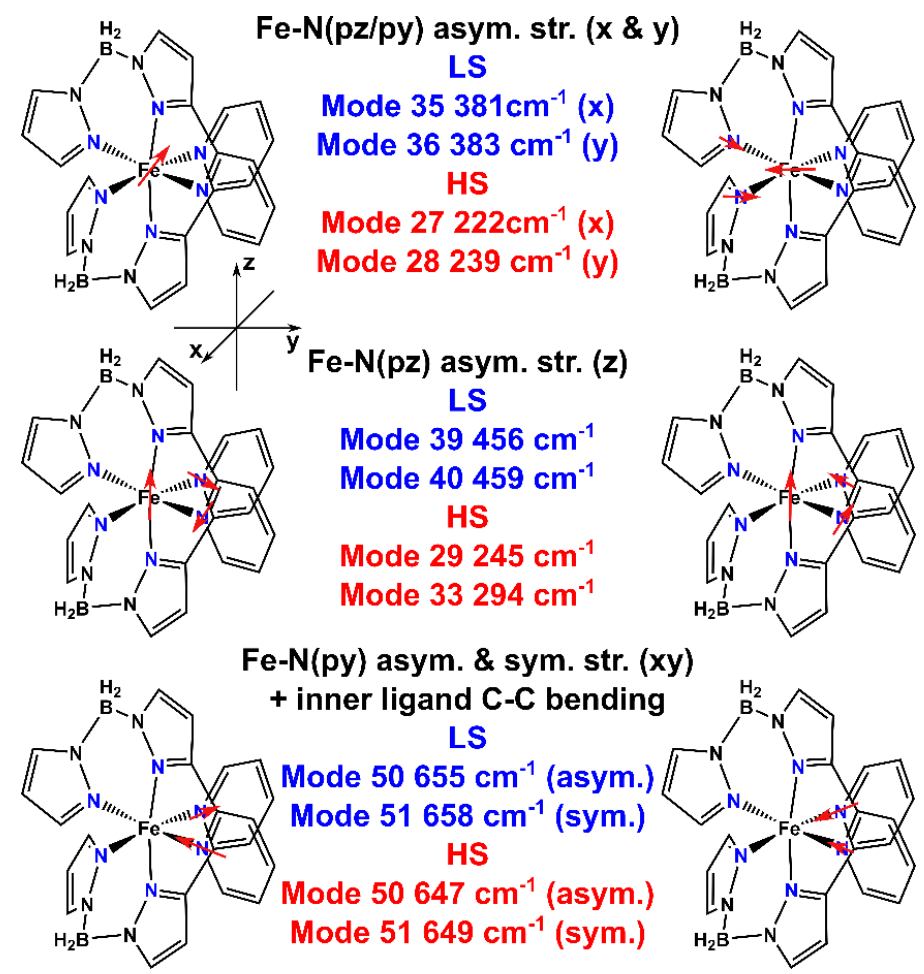

Figure S21. Selected and simplified metal-ligand stretching vibrations calculated for 1. The vibrational frequencies are obtained from DFT calculations (B3LYP/def2-SVP). The vectors are scaled according to the strength of the vibrational mode.

Table S3. Selected Far-Infrared vibrational and Resonance-Raman frequencies [ $\mathrm{cm}^{-1}$ ] for 1-II and 1-I. The vibrational frequencies obtained from DFT (B3LYP/def2-SVP) are shown in brackets and the measured frequencies are marked as bold. (Abbreviations: ip = in-plane; oop = out-of-plane; bend. = bendings; str. = strechting; $\mathrm{I}_{\text {calc. }}=$ calculated IR intensity or Raman activity).

\begin{tabular}{|c|c|c|c|c|c|c|c|c|}
\hline Mode & FIR HS & $\mathrm{I}_{\text {calc. }}$ & FIR LS & $\mathrm{I}_{\text {calc. }}$ & $\begin{array}{c}\text { Raman } \\
\text { HS }\end{array}$ & $\mathrm{I}_{\text {calc. }}$ & Raman LS & $\mathrm{I}_{\text {calc. }}$ \\
\hline $\begin{array}{c}\mathrm{Fe}-\mathrm{N}(\mathrm{pz} / \mathrm{py}) \\
\text { asym. str. (x \& y) }\end{array}$ & $\begin{array}{c}{\left[\begin{array}{c}x \\
222]\end{array}\right.} \\
\&[y \\
239]\end{array}$ & $\begin{array}{c}\text { x } 25.01 \\
\& \mathrm{y} \\
35.11\end{array}$ & $\begin{array}{c}{\left[\begin{array}{ll}\mathrm{x} & 381] \\
\& & 397[y \\
383]\end{array}\right.} \\
\end{array}$ & $\begin{array}{c}\text { x } 1.06 \\
\& y \\
1.76\end{array}$ & 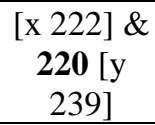 & $\begin{array}{c}\text { x } 0.61 \\
\& y \\
7.78\end{array}$ & $\begin{array}{c}\text { [x 381] \& } \\
393 \text { [y } \\
383]\end{array}$ & $\begin{array}{c}\text { x } 2.57 \\
\& y \\
1.96\end{array}$ \\
\hline $\begin{array}{c}\mathrm{Fe}-\mathrm{N}(\mathrm{pz}) \text { asym. } \\
\text { str. (z) }\end{array}$ & $\begin{array}{c}{[245] \&} \\
{[294]}\end{array}$ & $\begin{array}{c}10.20 \& \\
19.72\end{array}$ & $\begin{array}{c}\mathbf{4 4 1} \\
{[456] \&} \\
\mathbf{4 5 7} \\
{[459]} \\
\end{array}$ & $\begin{array}{c}1.51 \\
\& \\
0.34\end{array}$ & $\begin{array}{c}247[245] \\
\&[294]\end{array}$ & $\begin{array}{c}8.80 \& \\
9.38\end{array}$ & $\begin{array}{c}\mathbf{4 3 7}[456] \\
\& \mathbf{4 5 4} \\
{[459]}\end{array}$ & $\begin{array}{c}0.23 \& \\
3.49\end{array}$ \\
\hline $\mathrm{BH}_{2}$ ip bend. & $\begin{array}{c}{[322 \&} \\
325]\end{array}$ & $\begin{array}{c}4.21 \& \\
3.79\end{array}$ & $\begin{array}{c}\mathbf{3 1 8} \\
{[324]}\end{array}$ & 1.96 & $\begin{array}{c}331[322 \\
\& 325]\end{array}$ & $\begin{array}{c}4.17 \& \\
4.19 \\
\end{array}$ & $\begin{array}{c}\mathbf{3 3 0 / 3 3 6} \\
{[324]}\end{array}$ & 3.54 \\
\hline $\mathrm{BH}_{2}$ ip bend. & [361] & 7.31 & - & - & 374 [361] & 3.87 & - & - \\
\hline $\begin{array}{l}\text { Mixed asym./sym. } \\
\text { ip bend. }\end{array}$ & - & - & $\begin{array}{l}\mathbf{4 1 0}[405 \\
\& 406]\end{array}$ & $\begin{array}{c}2.11 \\
\& \\
2.84\end{array}$ & - & - & $\begin{array}{c}{[405 \&} \\
406]\end{array}$ & $\begin{array}{c}3.00 \& \\
6.20\end{array}$ \\
\hline
\end{tabular}




\begin{tabular}{|c|c|c|c|c|c|c|c|c|}
\hline Fe-N(py) asym. \& & {$[647 \&$} & $4.60 \&$ & {$[655 \&$} & 0.55 & $\mathbf{6 2 7}[647$ & $4.29 \&$ & $\mathbf{6 3 5}[655$ & 28.73 \\
sym. str. (xy)+ C- & 649] & 3.79 & $658]$ & $\begin{array}{c}\text { \& } \\
\text { C bend. }\end{array}$ & $\begin{array}{c}\text { \& 649] } \\
12.37\end{array}$ & $\begin{array}{c}\text { \& 658] } \\
\text { \& }\end{array}$ & \begin{tabular}{c} 
26.20 \\
\hline
\end{tabular} \\
\hline
\end{tabular}

\section{Investigations of vacuum-deposited films}
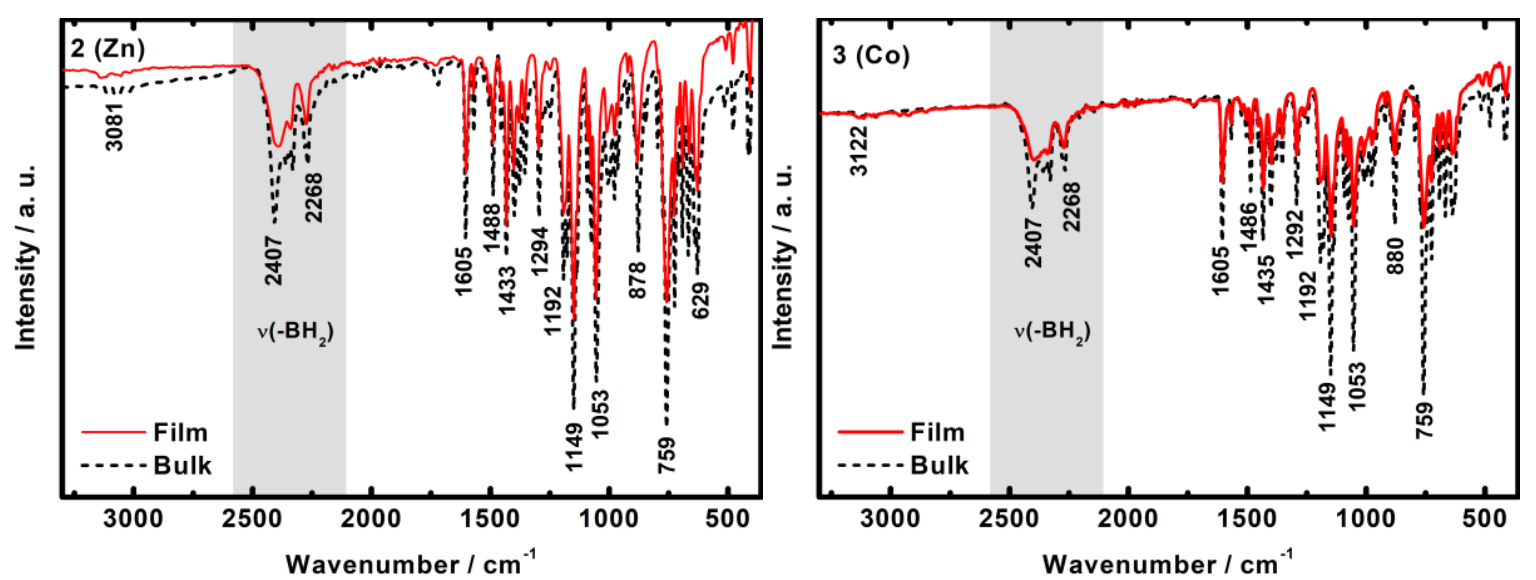

Figure S22. Fourier transform infrared (FT-IR) spectra of bulk material (black line) and vacuum-deposited material (red line) of $\mathbf{2}$ (left) and $\mathbf{3}$ (right) at $298 \mathrm{~K}$.

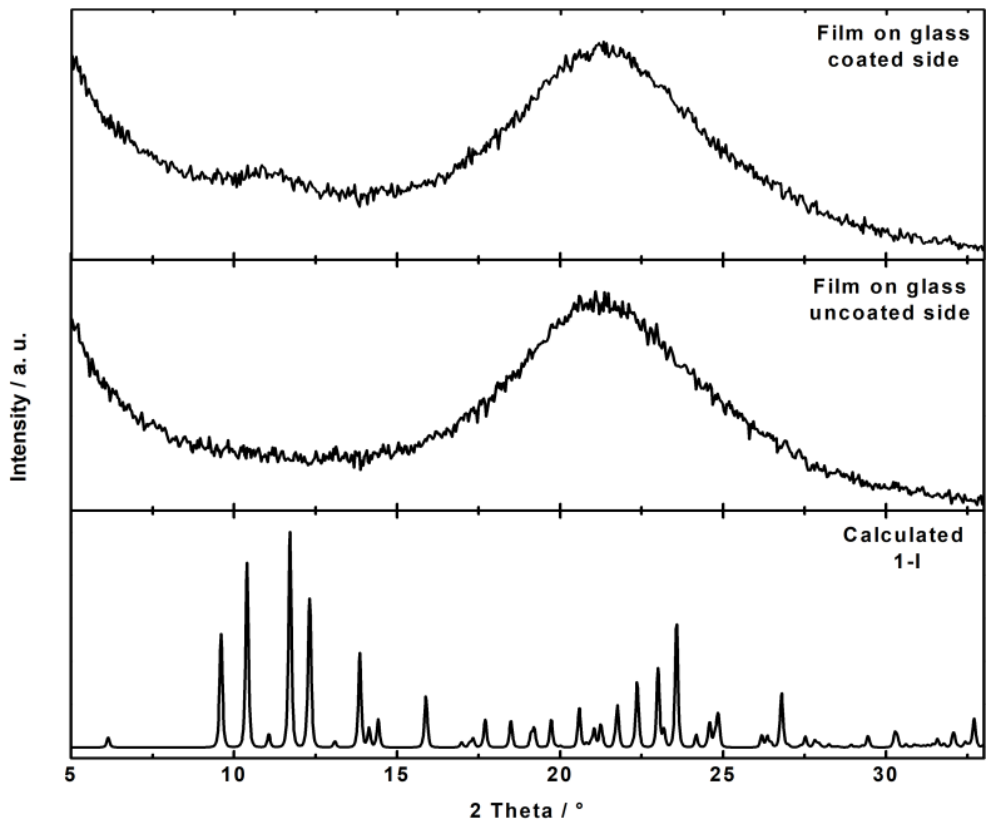

Figure S23. Experimental X-ray-diffractograms of film on glass for 1 with the coated side (top) and the uncoated side (middle) compared with the calculated XRPD pattern of 1-I. 


\section{Crystal structures and temperature dependent measurements}
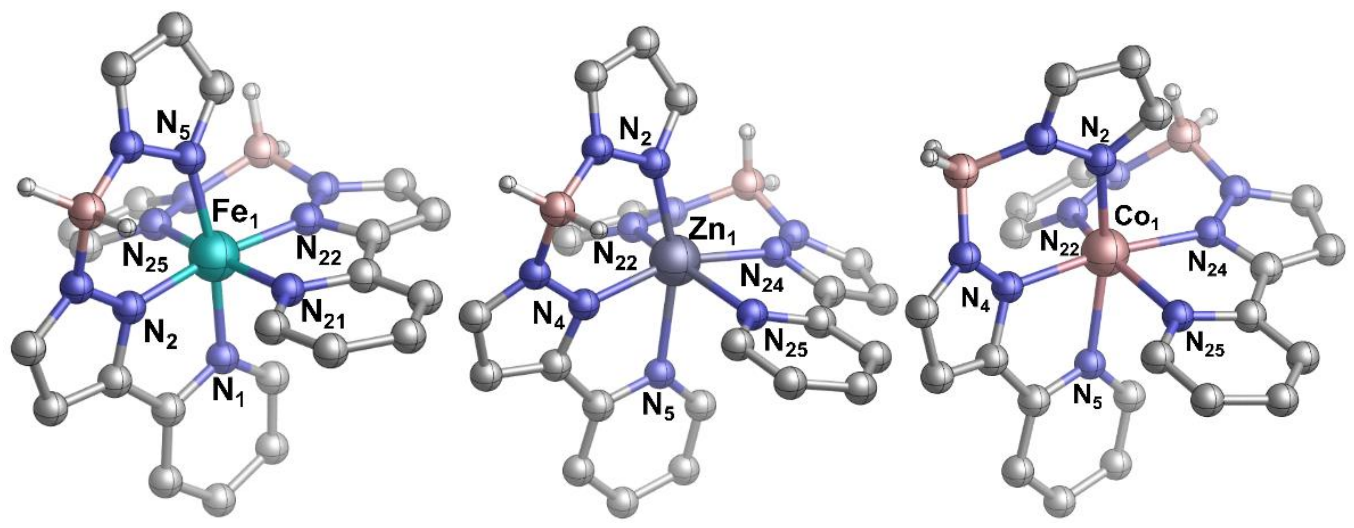

Figure S24. View of the asymmetric unit of 1-II (left), 2 (middle) and 3-MeOH (right). C-H hydrogen atoms and methanol molecules (for $\mathbf{3}$ ) are omitted for clarity and only selected atoms are labelled. Color code: $\mathrm{C}$ (grey), $\mathrm{H}$ (white), B (pink), N (blue), Fe (cyan), Zn (purple) and Co (pink).

Table S4. Selected bond lengths $[\AA]$ and angles $\left[{ }^{\circ}\right]$ for $\mathbf{1 - I I}$ at $300,393,423 \mathrm{~K}$ and $300 \mathrm{~K}$ after cooling from $393 \mathrm{~K}$.

\begin{tabular}{|l|l|l|l|l|l|}
\hline Bond & $\mathbf{2 0 0} \mathbf{K}$ & $\mathbf{3 0 3} \mathbf{K}$ & $\mathbf{3 9 3} \mathbf{K}$ & $\mathbf{4 2 3} \mathbf{K}$ & $\begin{array}{c}\text { 300 K } \\
\text { (after cooling from 393 K) }\end{array}$ \\
\hline Fe1-N1(py) & $2.0013(14)$ & $2.003(2)$ & $2.077(2)$ & $2.181(8)$ & $2.002(2)$ \\
\hline Fe1-N21(py) & $1.9919(14)$ & $1.997(1)$ & $2.059(2)$ & $2.153(8)$ & $1.997(1)$ \\
\hline Fe1-N2(pz) & $1.9031(14)$ & $1.909(2)$ & $1.951(2)$ & $2.01(1)$ & $1.908(2)$ \\
\hline Fe1-N22(pz) & $1.9108(14)$ & $1.915(2)$ & $1.960(2)$ & $2.03(1)$ & $1.915(2)$ \\
\hline Fe1-N5(pz) & $1.9742(13)$ & $1.978(2)$ & $1.999(3)$ & $2.06(1)$ & $1.979(2)$ \\
\hline Fe1-N25(pz) & $1.9918(14)$ & $1.995(1)$ & $2.033(2)$ & $2.084(8)$ & $1.994(1)$ \\
\hline & & & & & \\
\hline Average bond length & $\mathbf{1 . 9 6 2 2}$ & $\mathbf{1 . 9 6 6}$ & $\mathbf{2 . 0 1 3}$ & $\mathbf{2 . 0 8 6}$ & $\mathbf{1 . 9 6 6}$ \\
\hline N2-Fe1-N21 & $92.91(6)$ & $93.17(7)$ & $93.43(9)$ & $93.3(4)$ & $90.55(6)$ \\
\hline N21-Fe1-N22 & $80.40(6)$ & $80.46(7)$ & $78.57(8)$ & $75.9(3)$ & $80.39(6)$ \\
\hline N22-Fe1-N25 & $91.94(6)$ & $91.67(7)$ & $90.62(9)$ & $87.9(4)$ & $91.86(6)$ \\
\hline N25-Fe1-N2 & $94.29(6)$ & $94.27(7)$ & $96.6(1)$ & $101.4(4)$ & $94.28(6)$ \\
\hline N1-Fe1-N22 & $93.63(6)$ & $93.72(7)$ & $94.18(8)$ & $94.2(3)$ & $93.78(6)$ \\
\hline N22-Fe1-N5 & $94.55(6)$ & $94.44(7)$ & $96.36(9)$ & $101.9(4)$ & $94.36(6)$ \\
\hline N5-Fe1-N2 & $91.89(6)$ & $91.75(7)$ & $91.25(9)$ & $87.9(4)$ & $91.93(6)$ \\
\hline N2-Fe1-N1 & $80.33(6)$ & $80.45(7)$ & $78.56(9)$ & $76.4(4)$ & $80.30(6)$ \\
\hline N5-Fe1-N25 & $90.65(6)$ & $90.69(7)$ & $91.9(1)$ & $93.8(4)$ & $90.67(6)$ \\
\hline N25-Fe1-N1 & $86.05(6)$ & $86.28(7)$ & $86.04(9)$ & $86.1(4)$ & $86.23(6)$ \\
\hline N1-Fe1-N21 & $90.66(6)$ & $90.55(7)$ & $89.43(8)$ & $87.4(3)$ & $90.55(6)$ \\
\hline N21-Fe1-N5 & $93.68(6)$ & $93.56(7)$ & $94.55(9)$ & $97.0(3)$ & $93.60(6)$ \\
\hline & & & & & \\
\hline
\end{tabular}


Table S5. Selected bond lengths $[\AA]$ and angles $\left[{ }^{\circ}\right]$ for $\mathbf{2}$ and 3-MeOH at $200 \mathrm{~K}$.

\begin{tabular}{|c|c|c|c|}
\hline Zn1-N25(py) & $2.3114(19)$ & Co1-N25(py) & $2.218(2)$ \\
\hline Zn1-N5(py) & $2.3320(19)$ & Co1-N5(py) & $2.253(2)$ \\
\hline Zn1-N24(pz) & $2.0659(19)$ & Co1-N24(pz) & $2.048(2)$ \\
\hline Zn1-N4(pz) & $2.067(2)$ & Co1-N22(pz) & $2.105(2)$ \\
\hline Zn1-N22(pz) & $2.1583(18)$ & Co1-N2(pz) & $2.110(2)$ \\
\hline Zn1-N2(pz) & $2.079(2)$ & Co1-N4(pz) & $2.055(2)$ \\
\hline Average bond length & 2.1689 & Average bond length & 2.1315 \\
\hline N2-Zn1-N4 & $89.37(9)$ & N5-Co1-N24 & $95.92(8)$ \\
\hline N4-Zn1-N5 & $73.19(8)$ & N5-Co1-N25 & $79.82(8)$ \\
\hline N5-Zn1-N24 & $91.59(7)$ & N5-Co1-N4 & $74.12(8)$ \\
\hline N24-Zn1-N2 & $106.64(8)$ & N5-Co1-N22 & $92.81(8)$ \\
\hline N24-Zn1-N22 & $88.41(7)$ & N2-Co1-N25 & $88.58(8)$ \\
\hline N22-Zn1-N4 & $101.03(7)$ & N2-Co1-N24 & $101.76(8)$ \\
\hline N4-Zn1-N25 & $92.50(7)$ & $\mathrm{N} 2-\mathrm{Co} 1-\mathrm{N} 22$ & $104.24(8)$ \\
\hline N25-Zn1-N24 & $74.46(7)$ & N2-Co1-N4 & $87.22(8)$ \\
\hline N22-Zn1-N5 & $82.62(7)$ & N4-Co1-N25 & $100.78(8)$ \\
\hline N5-Zn1-N24 & $91.59(7)$ & N25-Co1-N24 & $74.98(9)$ \\
\hline $\mathrm{N} 25-\mathrm{Zn} 1-\mathrm{N} 2$ & $99.83(7)$ & N24-Co1-N22 & $88.60(9)$ \\
\hline N2-Zn1-N22 & $95.48(8)$ & N22-Co1-N4 & $93.82(9)$ \\
\hline
\end{tabular}

$$
\Sigma=\sum_{i=1}^{12}\left(\left|\varphi_{i}-90\right|\right) \text { and } \Theta=\sum_{j=1}^{24}\left(\left|\theta_{j}-60\right|\right)
$$

Table S6. Structural parameters $\Sigma\left[{ }^{\circ}\right]$ and $\theta\left[{ }^{\circ}\right]$ used to characterize the distortion of the $\mathrm{FeN}_{6}$ octahedron for 1-II at 200, 303, 393 and $423 \mathrm{~K}, \mathbf{2}$ and 3-MeOH at $200 \mathrm{~K}$.

\begin{tabular}{|l|l|l|l|l|l|l|}
\hline & $\mathbf{1 - I I} \mathbf{2 0 0} \mathbf{K}$ & $\mathbf{1 - I I} \mathbf{3 0 3} \mathbf{~ K}$ & $\mathbf{1 - I I} \mathbf{3 9 3} \mathbf{~ K}$ & $\mathbf{1 - I I} \mathbf{4 2 3} \mathbf{~ K}$ & $\mathbf{2}$ & $\mathbf{3} \cdot \mathbf{2 . 5}$ methanol \\
\hline $\mathbf{\Sigma}$ & 47.43 & 46.63 & 56.28 & 79.91 & 92.12 & 96.06 \\
\hline $\boldsymbol{\theta}$ & 149.08 & 147.89 & 187.01 & 264.21 & 298.06 & 310.77 \\
\hline
\end{tabular}

Calculation of structural parameters used to characterize the distortion of the $\mathrm{FeN}_{6}$ octahedron (Table S3). $\Sigma$ is calculated from the 12 cis-N-Fe-N angles and is a general measure of the deviation of a metal ion from an ideal octahedral geometry. $\Theta$ is calculated from 24 unique $\mathrm{N}$ $\mathrm{Fe}-\mathrm{N}$ angles measured on the projection of two triangular faces of the octahedron along their common pseudo-threefold axis and indicates more specifically its distortion from an octahedral towards a trigonal prismatic structure. A perfectly octahedral complex gives $\Sigma=\Theta=0 .{ }^{7-9}$ These parameters can be calculated manually or with the program OctaDist. ${ }^{10}$ 


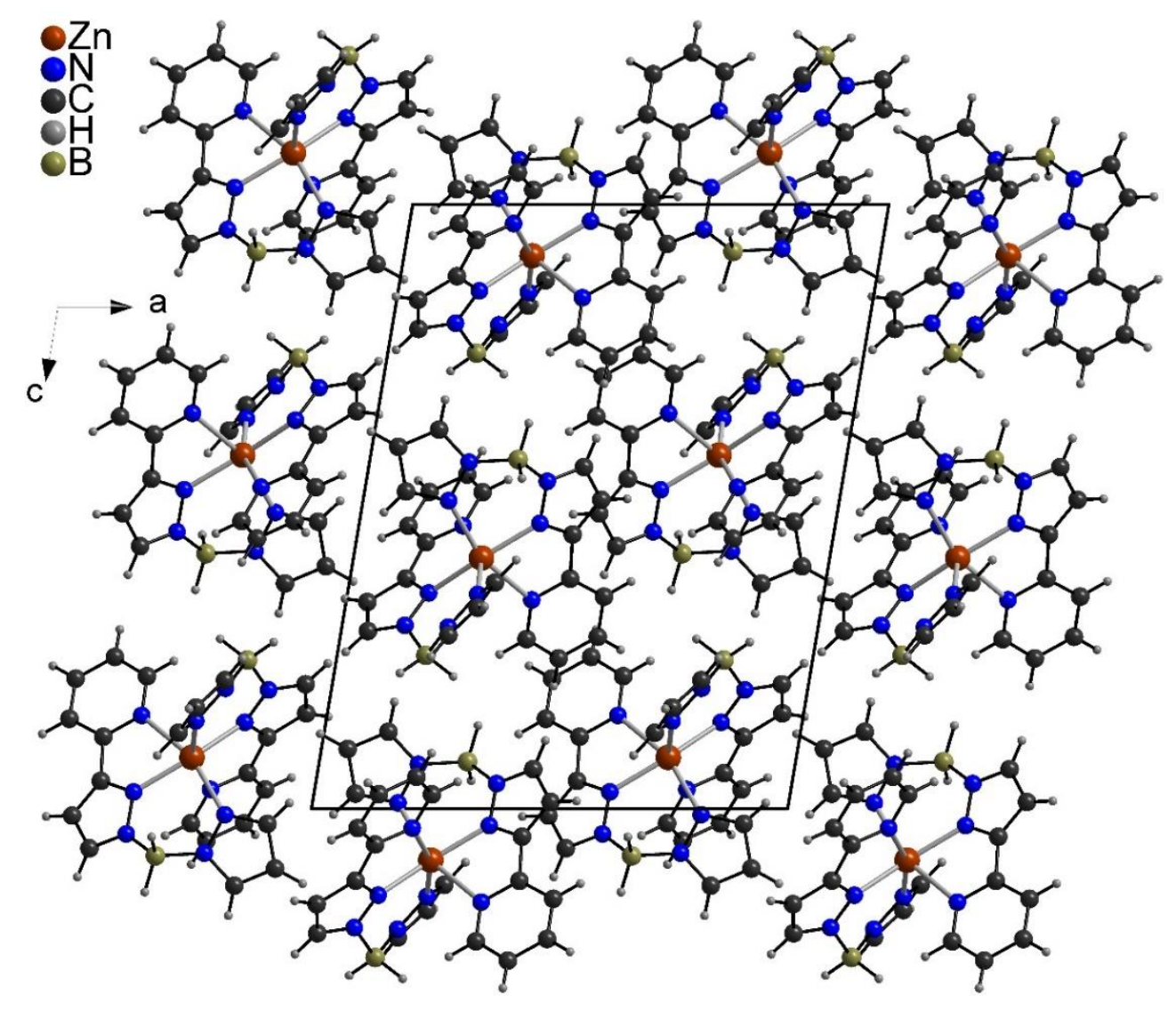

Figure S25. Crystal structure of $\mathbf{2}$ that is isotypic to 1-I with view along the crystallographic $b$-axis.

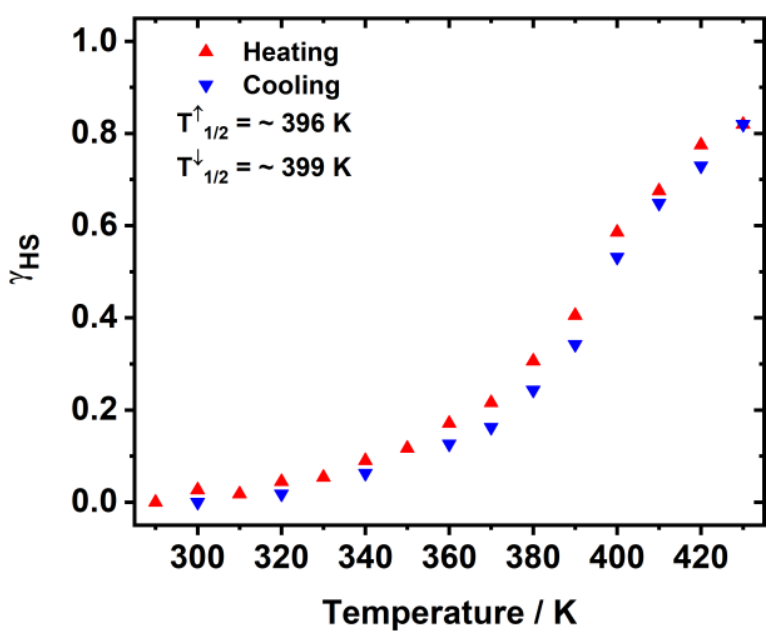

Figure S26. $\gamma_{H S}$ plot vs. $T$ obtained from temperature-dependent single crystal measurements of 1-II. 
Table S7. Temperature-dependent unit cell parameter for $\mathbf{1}$-II.

\begin{tabular}{|l|l|l|l|l|l|l|l|l|}
\hline $\mathbf{T}$ & $\mathbf{a} / \mathbf{A}$ & $\mathbf{b} / \mathbf{A}$ & $\mathbf{c} / \mathbf{A}$ & $\boldsymbol{\alpha} /{ }^{\circ}$ & $\boldsymbol{\beta} /{ }^{\circ}$ & $\boldsymbol{\gamma} /{ }^{\circ}$ & $\mathbf{V} / \AA^{\mathbf{3}}$ & $\mathbf{n R}$ \\
\hline $\mathbf{2 9 0}$ & 8.77 & 9.81 & 15.37 & 102.85 & 90.61 & 114.47 & 1164 & 400 \\
\hline $\mathbf{3 0 0}$ & 8.78 & 9.81 & 15.38 & 102.87 & 90.59 & 114.47 & 1167 & 387 \\
\hline $\mathbf{3 1 0}$ & 8.78 & 9.81 & 15.37 & 102.93 & 90.56 & 114.52 & 1166 & 377 \\
\hline $\mathbf{3 2 0}$ & 8.79 & 9.82 & 15.38 & 102.87 & 90.58 & 114.5 & 1169 & 358 \\
\hline $\mathbf{3 3 0}$ & 8.79 & 9.82 & 15.38 & 102.89 & 90.6 & 114.49 & 1170 & 345 \\
\hline $\mathbf{3 4 0}$ & 8.8 & 9.83 & 15.39 & 102.85 & 90.56 & 114.5 & 1174 & 338 \\
\hline $\mathbf{3 5 0}$ & 8.81 & 9.84 & 15.41 & 102.9 & 90.55 & 114.54 & 1177 & 320 \\
\hline $\mathbf{3 6 0}$ & 8.83 & 9.87 & 15.42 & 102.91 & 90.55 & 114.58 & 1183 & 297 \\
\hline $\mathbf{3 7 0}$ & 8.84 & 9.88 & 15.44 & 102.95 & 90.52 & 114.56 & 1188 & 274 \\
\hline $\mathbf{3 8 0}$ & 8.86 & 9.93 & 15.47 & 102.94 & 90.45 & 114.62 & 1198 & 266 \\
\hline $\mathbf{3 9 0}$ & 8.89 & 9.98 & 15.5 & 103.05 & 90.28 & 114.65 & 1209 & 233 \\
\hline $\mathbf{4 0 0}$ & 8.93 & 10.07 & 15.54 & 103.13 & 90.04 & 114.72 & 1229 & 207 \\
\hline $\mathbf{4 1 0}$ & 8.95 & 10.13 & 15.55 & 103.28 & 89.75 & 114.84 & 1239 & 184 \\
\hline $\mathbf{4 2 0}$ & 8.98 & 10.18 & 15.58 & 103.45 & 89.45 & 114.87 & 1250 & 180 \\
\hline $\mathbf{4 3 0}$ & 9 & 10.19 & 15.59 & 103.52 & 89.27 & 114.87 & 1255 & 159 \\
\hline $\mathbf{4 2 0}$ & 8.97 & 10.16 & 15.56 & 103.4 & 89.5 & 114.77 & 1245 & 164 \\
\hline $\mathbf{4 1 0}$ & 8.94 & 10.12 & 15.54 & 103.28 & 89.81 & 114.77 & 1236 & 174 \\
\hline $\mathbf{4 0 0}$ & 8.91 & 10.05 & 15.53 & 103.14 & 90.1 & 114.7 & 1223 & 195 \\
\hline $\mathbf{3 9 0}$ & 8.87 & 9.95 & 15.48 & 102.99 & 90.36 & 114.59 & 1202 & 240 \\
\hline $\mathbf{3 8 0}$ & 8.84 & 9.89 & 15.46 & 102.94 & 90.52 & 114.46 & 1191 & 271 \\
\hline $\mathbf{3 7 0}$ & 8.82 & 9.86 & 15.43 & 102.89 & 90.56 & 114.43 & 1182 & 267 \\
\hline $\mathbf{3 6 0}$ & 8.8 & 9.85 & 15.42 & 102.89 & 90.58 & 114.42 & 1178 & 274 \\
\hline $\mathbf{3 4 0}$ & 8.79 & 9.82 & 15.39 & 102.84 & 90.61 & 114.44 & 1171 & 300 \\
\hline $\mathbf{3 2 0}$ & 8.78 & 9.81 & 15.37 & 102.83 & 90.65 & 114.45 & 1166 & 306 \\
\hline $\mathbf{3 0 0}$ & 8.77 & 9.8 & 15.36 & 102.83 & 90.62 & 114.39 & 1164 & 327 \\
\hline
\end{tabular}

The obtained temperature-dependent unit cell volumes were converted to $\gamma_{H S}$ according to the following equation:

$$
\gamma_{H S}=\frac{V(T)-V(\mathrm{LS})}{V(\mathrm{HS})-V(\mathrm{LS})}
$$

with $V(T)$ as the measured unit cell volume $(V)$ at the temperature $(T), V(\mathrm{LS})$ as the unit cell volume of the LS state (here $290 \mathrm{~K}$ ) and $V(\mathrm{HS})$ as the volume corresponding to the HS. The crystal data of 1-II were calibrated to $\gamma_{H S}=0.82$ at $430 \mathrm{~K}$ from the SQUID data (Figure S26), leading to good agreement with the extrapolated spin transition curve (Figure 15). 


\section{Coordinates from the DFT calculations of 1}

\section{Coordinates from ORCA-job of $\mathbf{1}$ in the HS state}

Fe 4.96956534211590

N 2.83644759662401

C 2.44864850048595

C 1.33628810146137

H 1.04318058125290

C 0.62873048979891

H $\quad-0.23837391518348$

C 1.04747886482888

H 0.52695056224547

C 2.16248318715280

H 2.53095159233221

C 3.27455288902346

$\mathrm{N} 4.34227221880106$

N 4.96025777990290

C 4.30781673363237

H 4.66379040543500

C 3.20992263505874

H 2.47826024682591

B 6.13208282003920

H 5.66236282614219

H 6.66777776384373

N 7.17941343984182

N 6.88176110166577

C 8.03270883487509

H 8.02254129395346

C 9.10706646729159

H 10.16300776378223

C 8.51161226927158

H 8.94565333531548

N 3.72646736339150

C 3.31233519123449

C 2.45569039749764

H 2.13488649071700

C 2.03493832333459

H 1.36781437454370

C 2.47946195157516

H 2.17726658454944

C 3.32760684988288

H 3.70950992839432

C 3.81624604655298

$\mathrm{N} \quad 4.71331552845959$

N 5.00182862536392

C 4.30961653527824

H 4.42198156777934

C 3.52797065347538

H 2.84751130334104

B 6.08769189945071

H 7.17123038255975

H 6.05085360046184

N 5.75340869893925

N 5.59695348930597

C 5.48852595048198

H 5.34007994610112

C 5.57885497198719

H 5.53143261947484

C 5.74529561690534
0.39881437051239 1.01234536168610 0.21269441341868 0.52930440367435 $-0.13339510353244$ 1.69948770515821

1.96847609167399 2.52612096775508 3.45570852185296 2.13647547322060 2.75395364015040 $-0.97535548361819$ $-1.13684595112441$ $-2.26800926986811$ $-2.84724659336167$ $-3.78363268769162$ $-2.05381043594460$ $-2.23471301933830$ $-2.83046742835588$ $-3.20158377726803$ $-3.73430950223072$ $-1.70924533426110$ $-0.51747412350165$ 0.15440271374009 1.16443403848843 $-0.61917660948228$ $-0.36159354277593$ $-1.79431701628163$ $-2.68862333383042$ $-0.49395716593105$ 0.40501393881609 0.03496176442422 0.77932220553316 $-1.29111365227810$ $-1.60357440718044$ $-2.21353302451456$ $-3.26149365636496$ $-1.76467592716666$ $-2.44205747476124$ 1.76482598424343 1.94531765955682 3.24564581984676 3.92023509031626 4.99694914007482 3.00949777384359 3.22383447785568 3.75758777767989 3.31478555850223 4.97539937237187 3.24267384942292 1.93382765434370 1.84698695247724 0.87644529414702 3.12194800444959 3.38564598419846 3.97838000827808
10.67112659962041 11.58230104377036 12.59905174714861 13.39397398129655 14.20966007796095 13.12782637221552 13.73592767621838 12.08127220817830 11.84515806367582 11.33659849668399 10.51313728545822 12.81176064145563 12.01004024345627 12.35574715234406 13.39025116117844 13.81300080786887 13.72099186609518 14.50416422867810 11.48220436140313 10.41318130993538 12.09816963320660 11.23040948940639 10.65899076350310 10.50205805218454 10.09396977068735 10.96342945882122 10.97336271127403 11.42056658114593 11.86177653462160 8.98848104808869 8.06737889984214 7.02128693883255 6.29097914230239 6.93309962536368 6.12589124765294 7.88452859991310 7.84811884551080 8.89879739264332 9.66691984465764 8.26308625632180 9.24954357500617 9.32327873684641 8.37772246470551 8.27397514405043 7.66462022259363 6.84464943888839 10.32744378810259 9.97103522253659 10.33472917876274 11.75912515512180 12.06620586103082 13.39815610246498 13.86932835242070 13.97853871388318 15.03213554343935 12.89126370659303 


\section{$\begin{array}{llll}\text { H } & 5.87262738114430 & 5.05773450895090 & 12.84607463293165\end{array}$}

\section{Coordinates from ORCA-job of 1 in the LS state}

Fe 4.60596302855240

N 2.81243326577350

C 2.34228412446617

C 1.16859773948291

H 0.82355285211969

C 0.46924764567806

H -0.44705115063812

C 0.96260253668004

H 0.45262535526761

C 2.13728196079885

H 2.55947810358771

C 3.17543615921237

N 4.26374750945009

N 4.94937099903266

C 4.31747106348532

H 4.72351685154032

C 3.16859625648395

H 2.44434419689299

B 6.11116639175041

H 5.60175212234224

H 6.84267994275626

N 6.93150333575584

N 6.41171529311283

C 7.42249470315080

H 7.24441369006152

C 8.62748773855576

H 9.62016338475452

C 8.26481958133584

H 8.85650212269292

N 3.71720400570786

C 3.51807456486633

C 2.88159766672033

H 2.74028582601555

C 2.44640454540318

H 1.94986086900848

C 2.66090602972293

H 2.34566407192086

C 3.29883404126572

H 3.49542820264904

C 4.02302784497219

$\mathrm{N} \quad 4.66012987361726$

N 5.02169735584807

C 4.62885880818866

H 4.84507816517249

C 3.98331343117286

H 3.54371756562728

B 5.94986764873073

H 7.10962373222973

H 5.73875377071686

N 5.64285030907494

N 5.42172307232757

C 5.55732794768695

H 5.41091877196940

C 5.87188090599771

H $\quad 6.04174719345904$

C 5.91152355130347

H 6.13611742448905
0.47332137454541 1.09058386127409 0.32908710563133 0.66648678244765 0.03366280976786 1.81080361515189 2.09472326127549 2.59058912282845 3.49916706969788 2.19285285770461 2.78177816432162 $-0.82439308295043$ $-0.93647521917808$ $-2.01529787570558$ $-2.61731726847517$ $-3.52576271848229$ $-1.88925045962566$ $-2.09966789328068$ $-2.55121663417867$ $-3.18489134066221$ $-3.24764114954693$ $-1.36101859249338$ $-0.24939360485965$ 0.41119994506700 1.36564225045528 $-0.28297541577322$ $-0.00221930849643$ $-1.40032462612837$ $-2.23134418068348$ $-0.48897400164523$ 0.28135769038385 $-0.22239432024211$ 0.42508869419560 $-1.54585938733901$ $-1.96141330841578$ $-2.33133195477959$ $-3.37510705772785$ $-1.76153125775263$ $-2.34223634337144$ 1.63675090003035 1.87232567130690 3.15178127804755 3.76476033693512 4.81799713516714 2.83071267839732 2.99634622689539 3.70181621237655 3.47617525008422 4.89709255722444 2.99954084114412 1.67502502463142 1.43058805497224 0.42736798066263 2.61506369112059 2.74530433102787 3.58450940857554 4.64756681844648
10.83865800836027

11.57517935154124

12.60286047455726

13.28742922493442

14.10657183368973

12.91167656299237

13.43428318312048

11.86161216900588

11.53769562613628

11.22511580881854

10.41007229620817

12.89868291825104

12.11366321515599

12.47861515718717

13.51778529512627

13.95570687571273

13.82569108028636

14.60817939469977

11.57938912706744

10.65974657596449

12.26084588364802

11.00982956358066

10.43277086007782

9.85241258151933

9.36574733014110

10.04286466339262

9.70099550532994

10.78705565569084

11.16335706151630

9.30345965179486

8.19701687257242

7.05665854203398

6.18969877744234

7.05216441145574

6.17225294031994

8.18825401957668

8.22774748614995

9.28918866095366

10.18985618490656

8.34001515484240

9.50358443643587

9.54186315575011

8.39598463578234

8.23501272681958

7.58798943925626

6.60819887030180

10.66755898674158

10.34587964487534

10.79071435776396

12.02811095995329

12.21796654657426

13.52877623330853

13.91980098799001

14.21364512053458

15.27935514728731

13.21773571123628

13.26351905362777

S28 


\section{References}

(1) Naggert, H.; Bannwarth, A.; Chemnitz, S.; von Hofe, T.; Quandt, E.; Tuczek, F. First Observation of Light-Induced Spin Change in Vacuum Deposited Thin Films of Iron Spin Crossover Complexes. Dalton Trans. 2011, 40, 6364-6366. https://doi.org/10.1039/C1DT10651A.

(2) Naggert, H.; Rudnik, J.; Kipgen, L.; Bernien, M.; Nickel, F.; Arruda, L. M.; Kuch, W.; Näther, C.; Tuczek, F. Vacuum-Evaporable Spin-Crossover Complexes: Physicochemical Properties in the Crystalline Bulk and in Thin Films Deposited from the Gas Phase. J. Mater. Chem. C 2015, 3, 7870-7877. https://doi.org/10.1039/C5TC00930H.

(3) Ossinger, S.; Naggert, H.; Kipgen, L.; Jasper-Toennies, T.; Rai, A.; Rudnik, J.; Nickel, F.; Arruda, L. M.; Bernien, M.; Kuch, W.; et al. Vacuum-Evaporable Spin-Crossover Complexes in Direct Contact with a Solid Surface: Bismuth versus Gold. J. Phys. Chem. C 2017, 121, 1210-1219. https://doi.org/10.1021/acs.jpcc.6b10888.

(4) Ossinger, S.; Naggert, H.; Bill, E.; Näther, C.; Tuczek, F. Electronic Structure, Vibrational Spectra, and Spin-Crossover Properties of Vacuum-Evaporable Iron(II) Bis(Dihydrobis(Pyrazolyl)Borate) Complexes with Diimine Coligands. Origin of Giant Raman Features. Inorg. Chem. 2019, 58, 12873-12887. https://doi.org/10.1021/acs.inorgchem.9b01813.

(5) Ossinger, S.; Näther, C.; Tuczek, F. Spin-Crossover Behavior of Bis[Dihydrobis(4Methylpyrazol-1-Y1-Borate)]-(2,2'-Bipyridine)Iron and Analogous Complexes in the Bulk and in Thin Films: Elucidating the Influence of $\pi-\pi$-Interactions on the Type of Spin Transition. J. Phys. Condens. Matter 2020, 32, 094001. https://doi.org/10.1088/1361-648X/ab5776.

(6) Ossinger, S.; Kipgen, L.; Naggert, H.; Bernien, M.; Britton, A. J.; Nickel, F.; Arruda, L. M.; Kumberg, I.; Engesser, T. A.; Golias, E.; et al. Effect of Ligand Methylation on the Spin-Switching Properties of Surface-Supported Spin-Crossover Molecules. J. Phys. Condens. Matter 2020, 32, 114003. https://doi.org/10.1088/1361-648X/ab5c92.

(7) Iasco, O.; Boillot, M.-L.; Bellec, A.; Guillot, R.; Rivière, E.; Mazerat, S.; Nowak, S.; Morineau, D.; Brosseau, A.; Miserque, F.; et al. The Disentangling of Hysteretic Spin Transition, Polymorphism and Metastability in Bistable Thin Films Formed by Sublimation of Bis(Scorpionate) Fe(II) Molecules. J. Mater. Chem. C 2017, 5, 11067- 
11075. https://doi.org/10.1039/C7TC03276E.

(8) Guionneau, P.; Marchivie, M.; Bravic, G.; Létard, J.-F.; Chasseau, D. Structural Aspects of Spin Crossover. Example of the $\left[\mathrm{Fe}^{\mathrm{II}} \mathrm{L}_{\mathrm{n}}(\mathrm{NCS})_{2}\right]$ Complexes. In Spin Crossover in Transition Metal Compounds II; Topics in Current Chemistry; Springer Berlin Heidelberg, 2004; Vol. 234, pp 97-128. https://doi.org/10.1007/b95414.

(9) Halcrow, M. A. Structure:Function Relationships in Molecular Spin-Crossover Materials. In Spin-Crossover Materials; John Wiley and Sons Ltd: Oxford, UK, 2013; pp 147-169. https://doi.org/10.1002/9781118519301.ch5.

(10) OctaDist: A Tool for Calculating Distortion Parameters in Coordination Complexes. 